\title{
DESEMPENHO E PREVISÕES DE UM MODELO REGIONAL ESTATÍSTICO PARA A REGIÃO SUL DO BRASIL
}

\author{
L. P. Pezzi ${ }^{1}$, V. Ubarana ${ }^{2} \&$ C. Repelli ${ }^{2}$ \\ Received July 5, 1999 / Accepted May 25, 2000
}

A variabilidade climática sazonal e inter-anual afetam as atividades agrícolas, com conseqüências na produção de grãos da Região Sul do Brasil. Essa dependência dos fatores climáticos motivou o desenvolvimento de um modelo regional climático. Esse modelo é chamado de SIMOC (Sistema de Modelagem dos Oceanos) e foi adaptado para prever anomalias sazonais de precipitação. O SIMOC baseia-se em uma técnica estatística denominada de Análise de Correlações Canônicas (ACC). Os preditores para esse modelo são os campos de Temperatura da Superfície do Mar (TSM) em áreas sobre os oceanos Pacífico e Atlântico Tropical e o preditando é a precipitação sobre a Região Sul do Brasil. Os resultados do modelo, usando-se somente o Oceano Atlântico como preditor, pareceu influir mais na precipitação quando essa se afasta da condição inicial. O Oceano Pacífico previu melhor nos meses mais próximos a condição inicial, ao contrário do caso anterior onde usou-se o Atlântico como preditor. Em geral, o uso dos dois oceanos simultaneamente como campo preditor melhorou sensivelmente a precisão das previsões quando comparado com os casos dos oceanos individuais. Para o episódio ENOS de 1997-1998, o modelo reproduziu razoavelmente bem as anomalias de precipitação observadas, mostrando o potencial do SIMOC em previsões sazonais climáticas para a região Sul do Brasil.

Palavras-chave: Modelo estatístico; Previsão climática sazonal; Episódio ENSO 19971998.

FORECAST AND PERFORMANCE OF A STATISTICAL REGIONAL MODEL FOR SEASONAL CLIMATE PREDICTION IN SOUTHERN BRAZIL - Seasonal and interannual climate variability affects agricultural activities and, therefore, grain production in Southern Brazil. This dependence on climate has motivated the development of a seasonal climate forecast model, based on statistical techniques such as the Canonical Correlation Analysis (CCA). This model is called SIMOC (Oceanic Modelling Statistical System) and it was adapted to forecast seasonal anomalies of rainfall. The predictors for this model are sea surface temperature in areas of the Pacific and the Atlantic oceans, and the predictand is precipitation over Southern Brazil. The Atlantic Ocean, as a predictor, seems to affect more the prediction in the correlation analysis when the lag increases. Considering the Pacific Ocean, the best forecast was obtained when the lag was smaller and at the beginning of the run, in opposition to the Atlantic Ocean. In general, the use of the two oceans together seems to improve the precision of the predictions as compared to the individual ocean cases. For the ENSO episode of 1997-1998, the model reproduced reasonably well the observed rainfall anomalies, showing the usefulness of SIMOC in seasonal climate forecast for the Southern Brazil.

Key words: Statistical model; Seasonal climate forecast; ENSO episode1997-1998

\footnotetext{
${ }^{1}$ Centro de Previsão de Tempo e Estudos Climáticos (CPTEC/INPE)

Rod. Presidente Dutra, Km 40 - Cachoeira Paulista - SP - Brasil - 12630-000

Tel: 012 560-8400 - Fax:012 561-2835 - luciano@cptec.inpe.br

${ }^{2}$ The Weather Channel - Atlanta, GA, USA
} 


\section{INTRODUÇÃO}

O Brasil é um país de dimensões continentais composto por varias regiões que possuem condições climáticas muito distintas entre si e neste trabalho será enfocada a Região Sul do País, que compreende os Estados do Rio Grande do Sul (RS), Santa Catarina (SC) e Paraná (PR). Essa é uma das regiões com a maior densidade de cultivo agrícola do país e em média, tem condições climáticas favoráveis à agricultura e também à pecuária. Segundo o IBGE (1996, citado por Berlato \& Fontana (1997)), a produção média de grãos no período de 1993 a 1995 foi de 74 milhões de toneladas de grãos, o que pode ser entendido como $51 \%$ da produção nacional.

Porém, a variabilidade sazonal e anual de alguns parâmetros meteorológicos influenciam em muito no desenvolvimento e rendimento de determinadas culturas agrícolas. Dentre estas encontram-se as mais importantes dessa região: a soja, o milho, o arroz e o trigo.

O fenômeno ENOS tem impactos diretos sobre as atividades agrícolas da Região Sul e hoje em dia já existem indícios de que os impactos podem ser positivos ou negativos na produção agrícola. Para a análise dos impactos do El Niño, devem-se considerar alguns fatores como o período do ano em que se observa, calendário agrícola (semeadura ou colheita, que são consideradas as fases mais críticas), tipo de cultura e área da produção agrícola. O trigo, que é uma cultura de inverno, pode ter seu rendimento prejudicado devido ao excesso hídrico que normalmente ocorre associado à fase quente do ENOS. No episódio El Niño de 1997/ 98 até o dia 15 de novembro de 1997, que é exatamente o primeiro período em que o excesso de precipitação é sentido na Região Sul, perdeu-se aproximadamente 568 mil toneladas da safra agrícola da região. Desse total, $82 \%$ refere-se ao trigo que é a principal cultura de inverno e foi severamente castigado devido as intensas precipitações, ocorridas no período de maturação e colheita, outubro e novembro de 1997. Porém, analisando-se outras variedades agrícolas como a soja e o milho, culturas não irrigadas no verão, verifica-se que as mesmas podem ser beneficiadas na fase quente do ENOS devido ao excesso de precipitação (Berlato \& Fontana, 1997).

Os impactos do ENOS bem como os padrões de precipitação em várias partes do globo já vem sendo estudados há algum tempo para várias regiões do planeta. Já na década de 20, por exemplo, Sir Walker registrou a ocorrência da Oscilação Sul (OS), que é a componente atmosférica do fenômeno acoplado ENOS.
Mais recentemente, Ropelewski \& Halpert (1987) fizeram um trabalho de análise de impactos do ENSO para várias regiões do globo e apesar de contarem com muito poucos dados observados no Sul do Brasil os seus resultados sugerem que em anos de El Niño ocorre um excesso de precipitação em relação à normal climatológica a partir do mês de novembro do ano de surgimento do fenômeno e estendendo-se até o mês de fevereiro do ano seguinte ao surgimento do mesmo. Um estudo similar ao anterior analisando os impactos globais na precipitação foi feito por Ropelewski \& Halpert (1989) porém analisando-se os anos em que ocorreram a fase fria do ENOS (La Niña). Alguns resultados desse trabalho mostram que esses anos tendem a apresentar uma queda na quantidade de precipitação inferior à normal climatológica no período que se estende de junho a dezembro do ano em que a fase fria ocorre. Rao \& Hada (1990), Aceituno (1988), Studzinski (1995) e Diaz et al. (1998) mostraram que em anos de ENOS durante sua fase quente na Região Sul ocorre geralmente um excesso de precipitação no sul do Brasil, norte da Argentina e Uruguai. Na fase fria do ENOS (La Niña), ao contrario ocorre uma deficiência de precipitação.

Grimm et al. (1996; 1998) realizaram estudos similares àqueles de Ropelewski \& Halpert (1987, 1989), porém usando um conjunto de dados de precipitação bastante denso (250 estações) sobre a Região Sul do Brasil. Esse estudo reforçou a evidência da relação do excesso de precipitação dessa região com o fenômeno El Niño. Em várias localidades do Sul do Brasil os efeitos devido ao fenômeno El Niño já começam a ser notados em várias sub-regiões a partir da primavera do ano do fenômeno e que, em geral, o Sul do Brasil apresenta um forte e consistente padrão de anomalias de precipitação associados aos extremos de ENOS, mais forte que na Argentina e Uruguai.

Devido a esses fatores mencionados anteriormente percebe-se o quão importante é um melhor entendimento dos mecanismos climáticos que afetam a região, bem como o desenvolvimento de modelos capazes de realizar previsões climáticas sazonais para essa região. Tomadores de decisões poderão gerenciar melhor ações para minimizar os impactos negativos de situações desfavoráveis ou, por outro lado, maximizar o proveito em situações favoráveis com o auxílio de previsões climáticas confiáveis. Para o Brasil, as regiões com maior previsibidade climática sazonal são a Amazônia, principalmente o norte e leste desta grande área, o norte do Nordeste e, em menor grau, o sul do país. A previsibilidade climática é baixa no Sudeste, Centro-Oeste e sul do Nordeste (Carlos Nobre, 
comunicação pessoal).

Um estudo similar ao apresentado aqui foi feito anteriormente por Studzinski (1995) onde foram, também, investigadas as relações dos Oceanos Pacífico a Atlântico Tropicais com a precipitação na Região Sul do Brasil, porém naquele trabalho o enfoque foi de um estudo diagnóstico. O principal objetivo do presente trabalho é testar um modelo estatístico que foi adaptado para fazer previsões sazonais de anomalias de precipitação voltados para a Região Sul do Brasil. Utiliza-se como ferramenta o Sistema de Modelagem Estatística dos Oceanos (SIMOC - Repelli \& Nobre, 2000) enfatizando-se as características e potencialidades prognosticas do mesmo. O SIMOC consiste em um conjunto de rotinas baseados em uma sofisticada técnica estatística que é o método de Análise de Correlações Canônicas (ACC). Esta é uma eficiente técnica de correlação multivariada que aplica-se entre campos ou variáveis físicas com o objetivo de encontrar algum tipo de correlação entre elas.

O SIMOC esta sendo utilizado operacionalmente no Centro de Previsão de Tempo e Estudos Climáticos (CPTEC) para prever-se anomalias de precipitação nas regiões Nordeste e Sul do Brasil. Também é utilizada uma outra versão do modelo para a previsão de anomalias de TSM do Oceano Atlântico Tropical. Experiências bem sucedidas da aplicação do SIMOC para estudos diagnósticos e previsões das anomalias de precipitação no nordeste do Brasil (Ubarana, 1996), na Venezuela (Rojas, 1997), em algumas regiões do Cone Sul que compreendem o Sul do Brasil (Pezzi, 1998), Uruguai e norte de Argentina (Ghietto, 1998) e de anomalias de TSM no Oceano Atlântico Tropical (Pezzi et al., 1998; Repelli \& Nobre, 2000) indicam o potencial do uso deste modelo para previsão sazonal do clima e estudos diagnósticos.

\section{DADOS E EXPERIMENTOS}

\section{O Preditor}

Os dados usados como preditor nesse trabalho são médias mensais da Temperatura da Superfície do Mar (TSM) dos Oceanos Pacífico e Atlântico Tropical, durante o período de 1950 a 1992 . A área total do preditor abrange os domínios tropicais e uma parte extra-tropical dos Oceanos Pacífico e Atlântico e está compreendida entre as latitudes $40^{\circ} \mathrm{S}$ e $30^{\circ} \mathrm{N}$, longitudes $120^{\circ} \mathrm{E}$ e $15^{\circ} \mathrm{W}$, como mostrado na Fig. 1 . Os campos de TSM são dados reconstruídos na resolução espacial

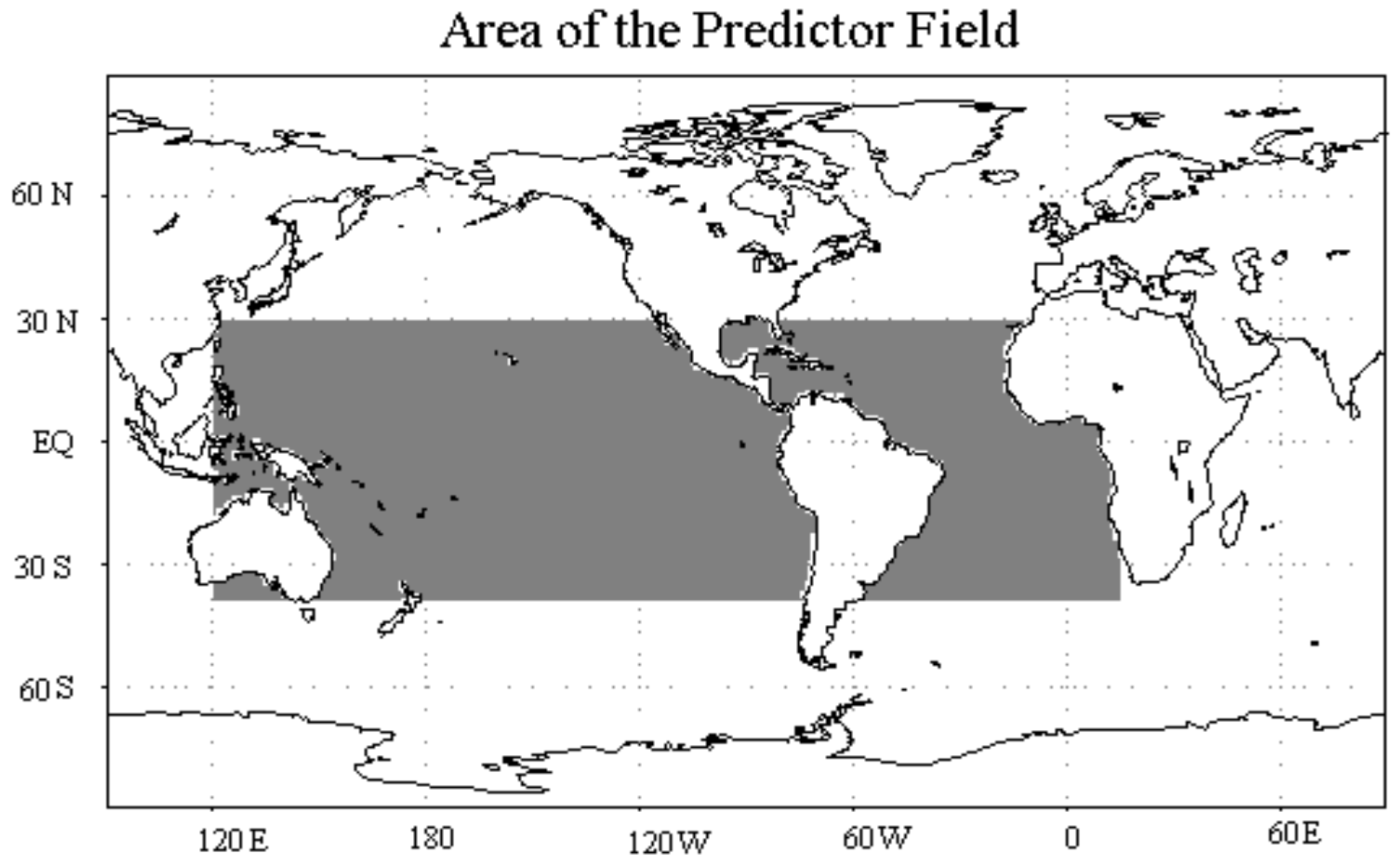

Figura 1 - Áreas das Temperaturas da Superfície do Mar (TSM) usadas como preditores pelo SIMOC para a previsão sazonal de anomalias de precipitação sobre a Região Sul do Brasil. Oceanos Pacífico e Atlântico Tropical (área sombreada).

Figure 1 - Areas of the Pacific and Atlantic Oceans (shaded areas) where Sea Surface Temperatures (SST) anomalies were defined to be used as predictor for SIMOC's seasonal rainfall anomalies forecast in Southern Brazil. 
de $1^{\circ} \times 1^{\circ}$, latitude e longitude respectivamente, e foram gerados pelo National Center for Environmental Prediction (NCEP/NOAA). São descritos com detalhes em Reynolds \& Smith (1995) e Smith et al. (1996). Essa base de dados é gerada usando-se o método de Interpolação Ótima (IO) espacial a partir de Funções Ortogonais Empíricas (FOE) construídas com dados de TSM do período base de 1982 a 1993. Com os modos dominantes espaciais e temporais das FOE, gerados a partir da OI, reconstruiu-se os campos globais de TSM. Segundo Smith et al. (1996), esse novo conjunto de TSM reconstruída apresenta erros médios quadrados (RMS) menores em relação aos dados in situ. Isto se deve ao fato de que a partir de novembro de 1981 foram introduzidas informações de satélite na geração das TSM, além dos dados in situ coletados por bóias e navios.

\section{O Preditando}

Como preditando foram utilizados totais mensais de precipitação da Região Sul do Brasil obtidos através de 51 estações meteorológicas de superfície. A série completa desses dados cobre o período de janeiro de 1950 a dezembro de 1992, perfazendo um total de 43 anos. Os dados são oriundos de várias fontes, Instituto Nacional de Meteorologia (INMET), Departamento Nacional de Energia Elétrica (DNAEE), Instituto de Pesquisas Agronômicas (FEPAGRO), Companhia Paranaense de Energia Elétrica (COPEL), Sistema Meteorológico do Paraná (SIMEPAR) e ITAIPU BiNacional. Os dados omissos dessa série foram preenchidos usando-se sempre o valor climatológico correspondente a respectiva série temporal por estação. Em todos os casos não foram usados estações onde os dados omissos ultrapassaram $20 \%$ do número total de dados que compuseram a série. As séries de dados do preditando abrangem os estados do Rio Grande do Sul, Santa Catarina e Paraná que juntos formam a Região Sul do Brasil. Essa área está compreendida entre as latitudes de $34^{\circ} \mathrm{S}$ e $22^{\circ} \mathrm{S}$ e longitudes de $58^{\circ} \mathrm{W}$ e $48^{\circ} \mathrm{W}$, como mostrado na Fig. 2.

\section{Os Grupos de Experimentos}

Foram realizados três grandes grupos com 144 experimentos cada um. Cada experimento consistiu em usar-se 12 vezes o mesmo preditor para 12 preditandos diferentes, defasados no tempo. O campo preditor foi usado em escala mensal sendo o mês de janeiro o primeiro e o mês de dezembro o último. O preditando
Area and Stations of the Predictand Field

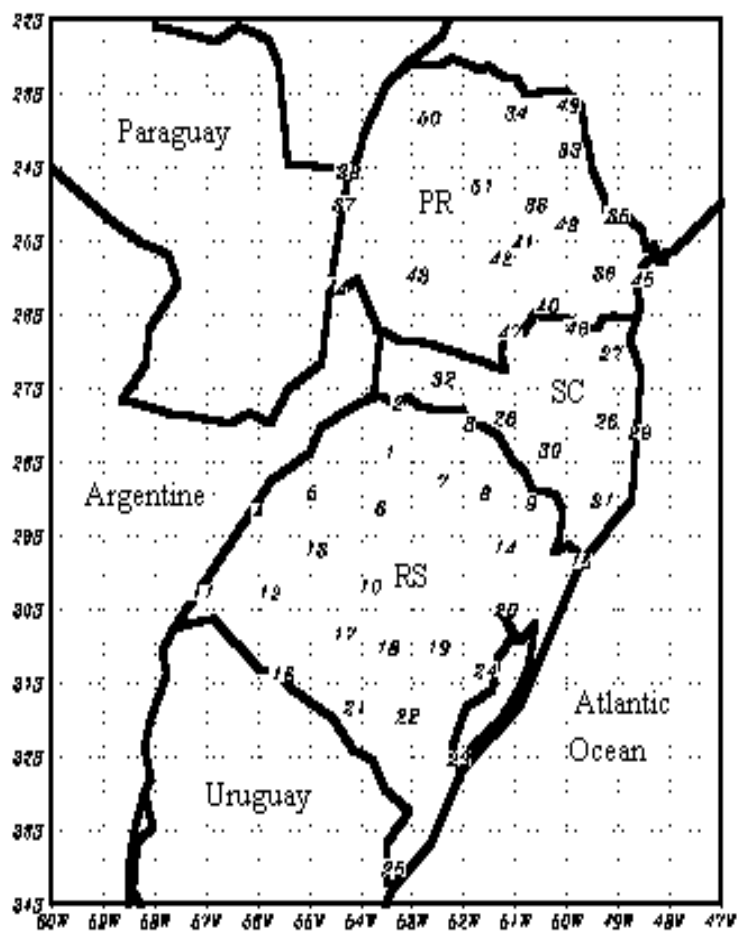

Figura 2 - Localização das 51 estações meteorológicas usadas como preditando pelo SIMOC para a previsão sazonal de anomalias de precipitação sobre a Região Sul do Brasil.

Figure 2 - Location of the 51 meteorological stations used as predictand for SIMOC's seasonal rainfall anomalies forecast in Southern Brazil.

foi tomado em escala trimestral sendo o primeiro trimestre janeiro, fevereiro, março (JFM) e o último dezembro, janeiro, fevereiro (DJF). Em cada um dos três grupos usou-se três áreas distintas dos campos preditores (TSM). No primeiro grupo usou-se como área preditora o domínio do Oceâno Atlântico Tropical $\left(40^{\circ} \mathrm{S}-30^{\circ} \mathrm{N}, 70^{\circ} \mathrm{W}-15^{\circ} \mathrm{E}\right)$. No segundo, tomou-se como área do preditor somente o Oceâno Pacífico Tropical $\left(40^{\circ} \mathrm{S}-30^{\circ} \mathrm{N}, 120^{\circ} \mathrm{E}-70^{\circ} \mathrm{W}\right)$ e no terceiro, dos oceânos Pacifico e Atlântico Tropical $\left(40^{\circ} \mathrm{S}-30^{\circ} \mathrm{N}, 120^{\circ} \mathrm{E}-15^{\circ} \mathrm{E}\right)$ simultaneamente. Em todos os casos usou-se o período de 1950 a 1980 na construção do modelo, totalizando 31 anos.

\section{O modelo SIMOC}

O modelo SIMOC usado nesse trabalho é o mesmo descrito em Repelli \& Nobre (2000) e foi construído usando o método de Análise de Correlações Canônicas (ACC). O método de ACC não foi apresentado nesse trabalho em termos de sua formulação matemática uma vez que a mesma pode ser encontrada em vários trabalhos já publicados. A 
descrição do método bem como a sua formulação matemática é mostrada em detalhes por Barnett \& Preisendorfer (1987) e também em Wilks (1995). Aplicações do método de ACC podem ser encontradas em outros trabalhos tais como Graham et al. (1987a, 1987b), Barnston \& Ropelewski (1992), Barnston (1994), Studzinski (1995), Diaz et al. (1998) Barnston \& Smith (1996), Pezzi et al. (1998) e Repelli \& Nobre (2000).

Em linhas gerais, a ACC é uma forma de regressão linear múltipla que identifica os padrões dominantes em cada um dos dois grupos multivariados de dados que, dentre todos os grupos multivariados, estejam mais correlacionados entre si. Assim, teoricamente, a partir de dois campos físicos $\mathbf{Y}(\mathbf{n t}$,ny) chamado de preditor e $\mathbf{Z}(\mathbf{n t}, \mathbf{n z})$ de preditando (onde nt representa as observações ao longo do tempo e ny e $\mathbf{n z}$ representam os pontos no espaço) podem ser encontrados dois vetores $\mathbf{r}$ e $\mathbf{q}$, com dimensões espaciais de modo que $\mathbf{u}(\mathbf{t})=\mathbf{Y r}$ e $\mathbf{v}(\mathbf{t})=\mathbf{Z q}$. Resolver o problema ACC consiste em encontrar $\mathbf{r}$ e $\mathbf{q}$ de modo que cada par de $\mathbf{u}(\mathbf{t})$ e $\mathbf{v}(\mathbf{t})$ possua correlação máxima entre si.

$\mathrm{Na}$ execução do modelo, antes de calcular-se a ACC, os campos do preditor (Y) e o preditando (Z) são primeiramente condensados separadamente usando Funções Ortogonais Empíricas (FOE). Esse procedimento equivale à passagem de um pré filtro e é realizado para remover-se a instabilidade e os ruídos das séries de dados. Por ser linear, a solução da ACC está sujeita à instabilidade, principalmente quando o número de observações no tempo não é muito grande, ou quando existem ruídos nas observações dos dados. A utilização de FOE reduz alguns dos ruídos aleatórios existentes. Desta forma, um número de modos de FOE são retidos de forma a explicar aproximadamente $80 \%$ total de cada série. A partir dos campos de TSM e precipitação, filtrados e reconstruídos com $\mathrm{FOE}$, é realizado o cálculo de ACC onde encontram-se as equações de previsão que relacionam a maximização da variância entre as variáveis preditora e preditanda.

Na Fig. 1 de Repelli (1995) é apresentado um fluxograma esquemático do SIMOC o qual mostra a lógica de funcionamento do sistema. O sistema permite que se escolha qualquer área do globo para ser usada como preditor e/ou preditando, e também que seja usado qualquer espaço de tempo de defasagem entre preditor e preditando que se queira. A leitura dos dados do preditor é feita sobre a área escolhida e os dados sobre o continente são extraídos através de máscaras. Após isso, calculam-se a climatologia, anomalias e o desvio padrão de TSM que é usado para normalizar-se as anomalias em cada ponto de grade. Para o preditando, que nesse caso é a precipitação, é feito um processo similar, apesar dessa variável encontrar-se em ponto estação.

\section{AVALIAÇÃO DO DESEMPENHO GERAL DO MODELO ESTATÍSTICO}

Com o objetivo de se ter uma idéia geral do desempenho do modelo, foram feitas previsões para anos passados, no período de 1981 a 1992 totalizando 12 anos de dados. Este período de dados observados é chamado de série independente de dados, uma vez que foram usados somente na verificação das previsões e não na construção do modelo. Para acessar o desempenho do modelo calculou-se os coeficientes de correlação médio das anomalias de precipitação sobre a área abrangida pelos três estados do Sul, entre os campos previstos e os observados, para esse período de 12 anos de dados. Calculou-se também, os coeficientes de correlação entre a persistência das anomalias de precipitação observadas no mês do preditor com as respectivas anomalias de precipitação observadas nos períodos dos preditandos. Com essas duas medidas pode-se avaliar em que época do ano e com quais áreas preditoras o desempenho do modelo foi melhor ou pior. Um teste estatístico de hipótesenula (t-student) foi aplicado para se determinar a significância das correlações obtidas (Bendat \& Person, 1986). De acordo com o resultado do teste estatístico aplicado, valores de correlações acima de 0,65 são significantes ao nível de $99 \%$ e valores de correlação maiores que 0,49 são significantes ao nível de 95\%.

Para se ter uma ilustração do desempenho do modelo em um caso específico, são apresentadas as previsões elaboradas pelo SIMOC para o período de primavera e verão do episódio ENOS dos anos 97/98. São mostradas as observações e as previsões sazonais feitas pelo modelo com uma antecedência de até 3 meses para esse período.

\section{Grupo 1 - Oceano Atlântico como Preditor}

A Fig. 3(a-1) mostra, para a Região Sul, as correlações entre as anomalias de precipitação previstas e observadas. Nesses mesmos gráficos são mostradas também as correlações das persistências das anomalias observadas de precipitação relativas ao período do preditor com as anomalias observadas referentes ao período do preditando. A influência do Oceano Atlântico parece aumentar com o aumento da defasagem da previsão, ou seja quanto mais afastada a previsão fica da condição inicial (preditor). Quando se usam os meses 
Correlation Coeff. Predutar jan (hi)

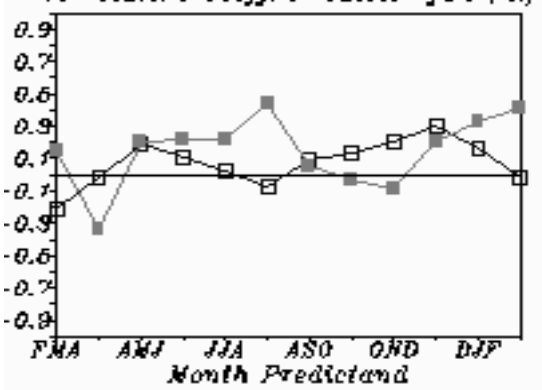

a)

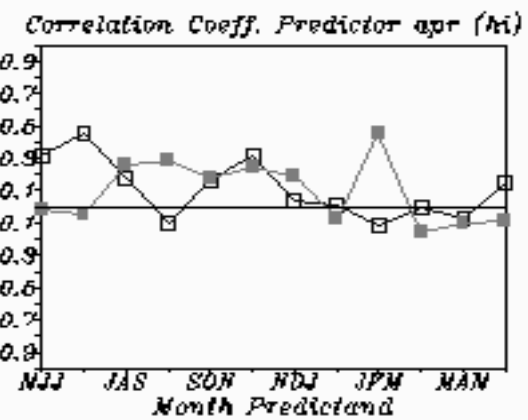

d)

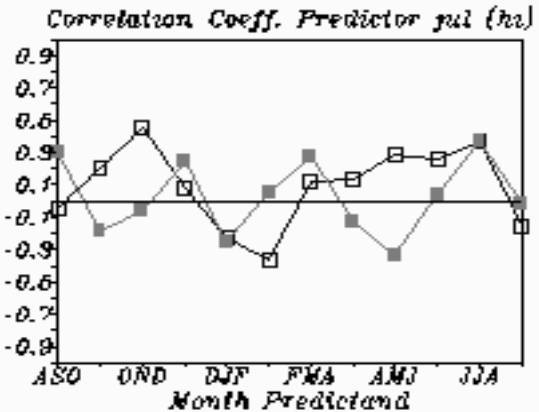

g)

Correlation coeff. Predtetor oct (hi)

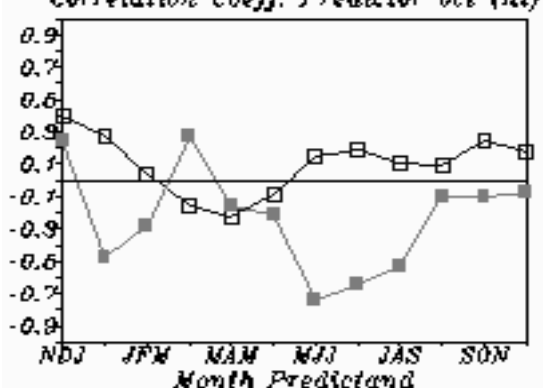

j)

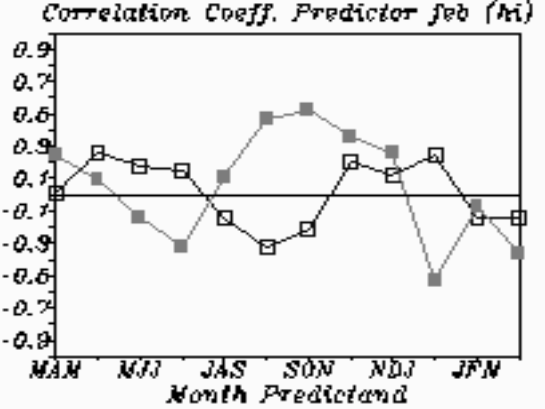

b)

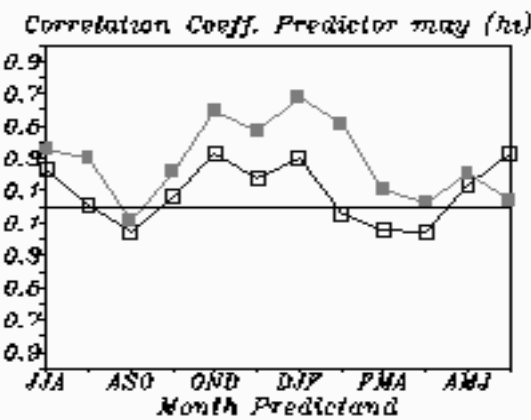

e)

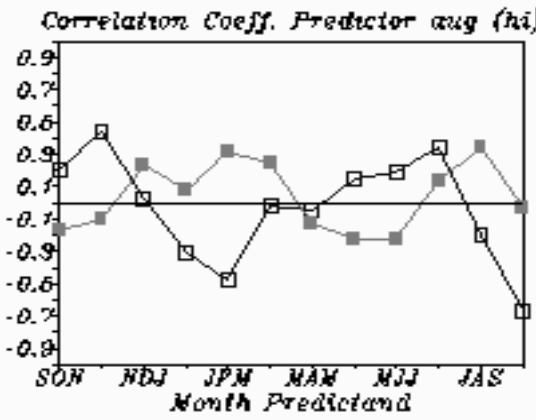

h)

Correlation Gobfs. Predictor now (ht)

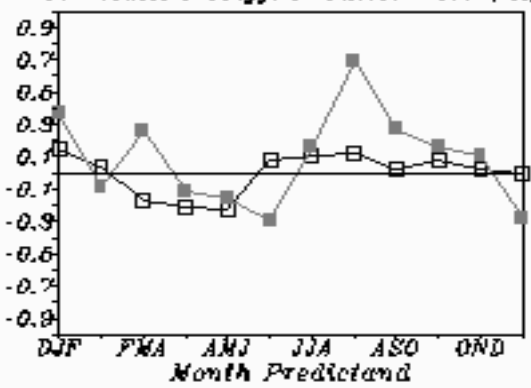

k)
Correlation coeff. Predictor mar (hi)

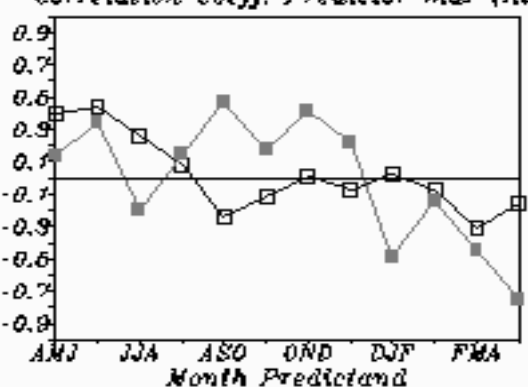

c)

Correlation Coeff. Predictor jun (hi)

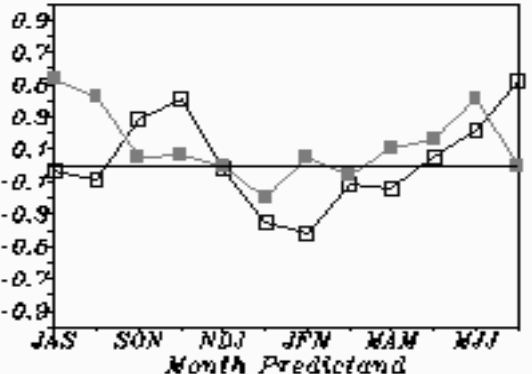

f)

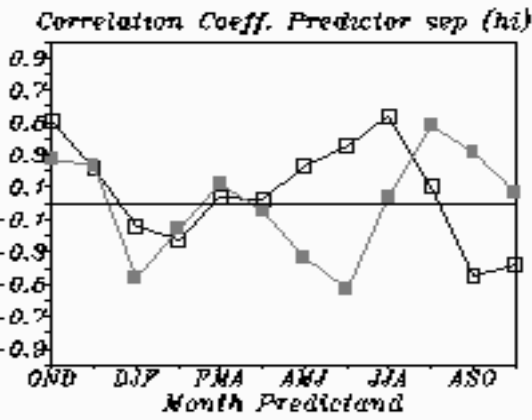

i)

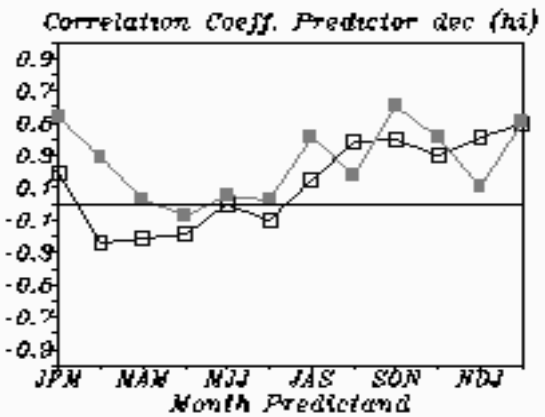

l)

Figura 3 - Coeficientes de correlação entre a persistência das anomalias de precipitação observadas com as anomalias observadas (símbolo aberto), e coeficientes de correlação entre as anomalias de precipitação previstas pelo SIMOC com as observadas (símbolo cheio), usando-se o Oceano Atlântico Tropical como campo preditor. Começando com o experimento feito em Janeiro (Fig. 3a) indo até Dezembro (Fig. 31). Cada experimento tem 12 meses de defasagem.

Figure 3 Correlation coefficients between observed precipitation anomalies and observed precipitation anomalies persistence (open symbols), and between forecasted precipitation anomalies and observed precipitation anomalies (full symbols), for SST in Tropical Atlantic Ocean as a predictor field. Initialization on the experiment starts from January (Fig. 3a) to December (Fig. 3l). Each experiment shown had a 12 month lag. 
Correlation Coeff. Predictor jan (hit)

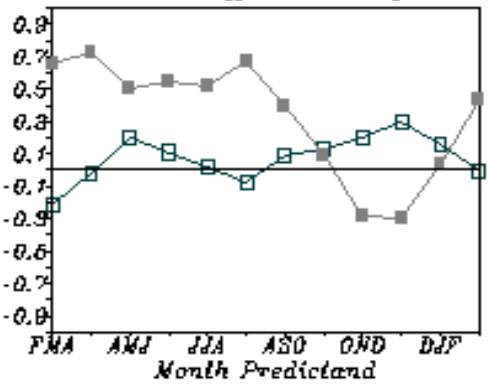

a)

Corelation Coeff. Predictor apr (hi)

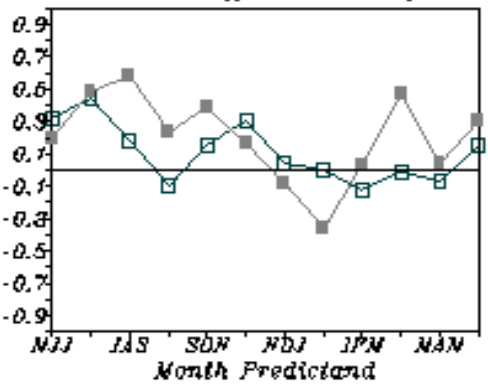

d)

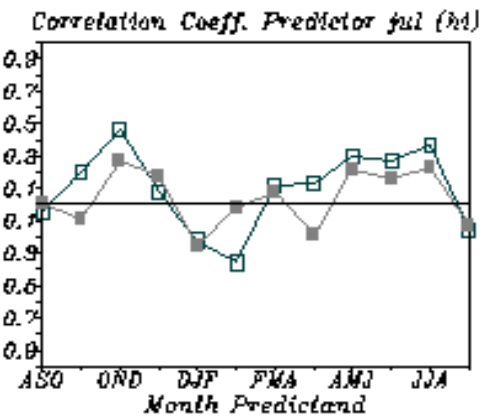

g)

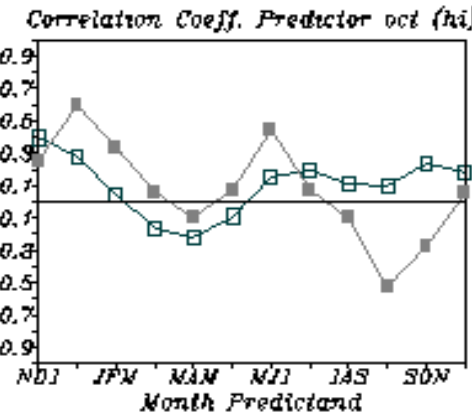

j)
Correlation Loeff. Predictor feb (Mi)

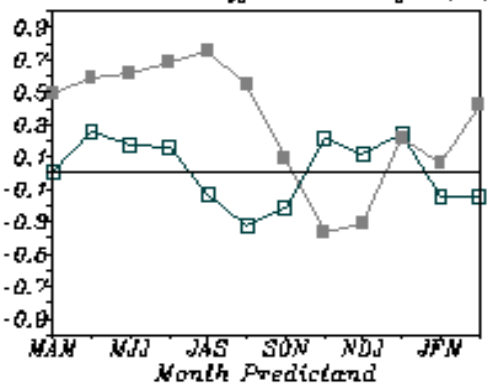

b)

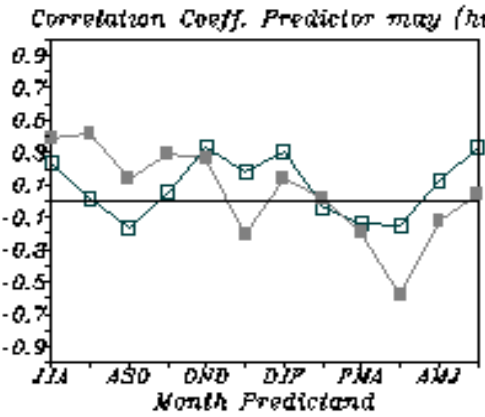

e)

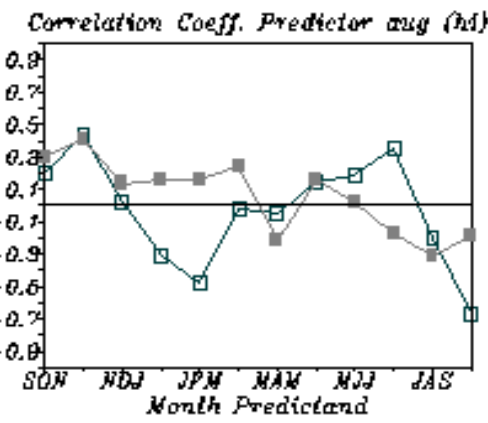

h)

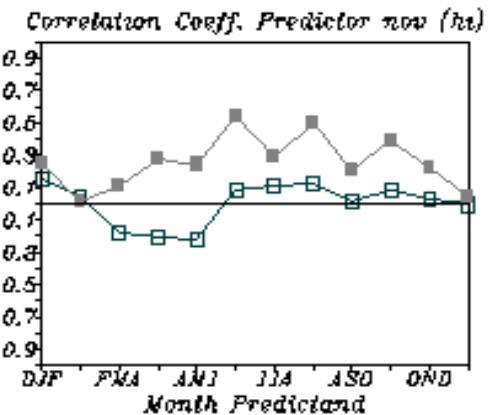

k)

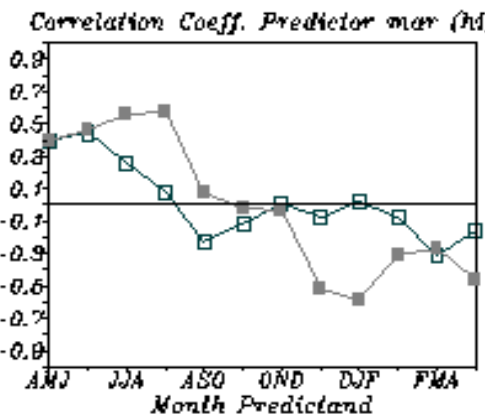

c)

Corelatim coeff. Predictor jun (hi)

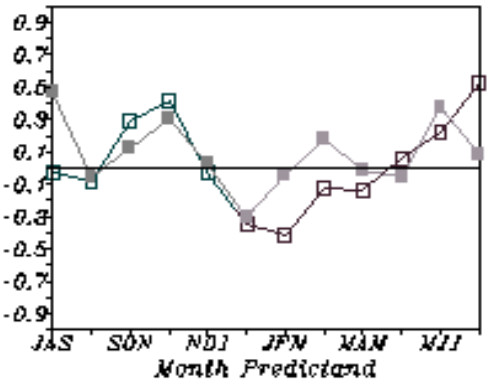

f)

Correlation Coeff Predictar sep oht

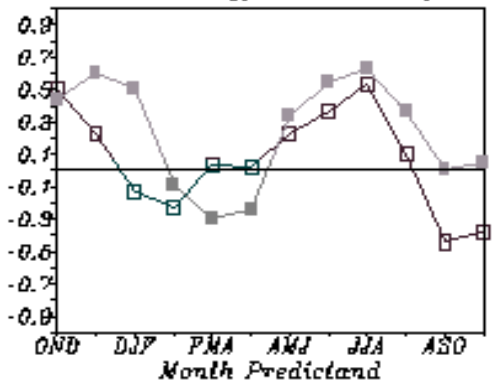

i)

Correiation coeff. Prodtctor dec (hi)

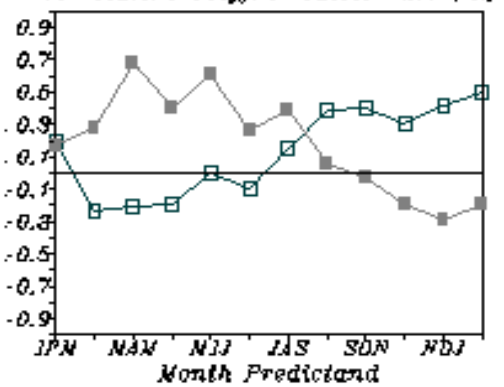

1)

Figura 4 - Coeficientes de correlação entre a persistência das anomalias de precipitação observadas com as anomalias observadas (símbolo aberto), e coeficientes de correlação entre as anomalias de precipitação previstas pelo SIMOC com as observadas (símbolo cheio), usando-se o Oceano Pacífico Tropical como campo preditor. Começando com o experimento feito em Janeiro (4a) indo até Dezembro (4l). Cada experimento tem 12 meses de defasagem.

Figure 4 Correlation coefficients between observed precipitation anomalies and observed precipitation anomalies persistence (open symbols), and between forecasted precipitation anomalies and observed precipitation anomalies (full symbols), for SST in Tropical Pacific Ocean as a predictor field. Initialization on the experiment starts from January (Fig. 4a) to December (Fig. 4 l). Each experiment shown had a 12 month lag. 
preditores de janeiro a maio (Fig. 3a a Fig. 3e), os primeiros trimestres previstos apresentam um baixo desempenho do modelo e a medida em que a previsão se afasta do mês do preditor o desempenho do modelo melhora. Com os meses de junho, novembro e dezembro (Fig. 3f, 3k e 3i, respectivamente), prevendo os demais trimestres, esse comportamento se inverte mostrando que o modelo tem um desempenho melhor do que a persistência para os dois, e em alguns casos três, primeiros trimestres de previsão em relação a esses meses preditores. Nos demais meses, os desempenhos do modelo usando-se somente o Atlântico como preditor foram muito baixos.

\section{Grupo 2 - Oceano Pacífico como Preditor}

Pela Fig. 4 (a-1) podemos ver que, em vários casos, os coeficientes de correlação para previsão são maiores que os de persistência, indicando com isto que a região apresenta uma certa previsibilidade. Exceção a isso, foram os meses de outubro (Fig. 4-j) e novembro (Fig. 4-k) prevendo o período seguinte. Os campos preditores do período de dezembro (Fig. 4-1) a setembro (Fig. 4-a a Fig. 4-i) demonstraram os melhores desempenhos sobre a área como um todo. Porém no período onde se usaram os campos de TSM de dezembro (Fig. 4-1) a maio (Fig. 4-e), pode-se afirmar que os melhores desempenhos do modelo foram obtidos para essa região. Os trimestres de maio, junho, julho (MJJ) e julho, agosto e setembro (JAS) são os períodos onde se verificaram as melhores correlações médias das previsões com as observações. Nestes casos os valores dos coeficientes de correlação variam entre 0,6 e 0,8. Esses dois períodos indicaram uma razoável previsibilidade com uma certa antecedência que no caso do trimestre JAS, chega a ser de até 6 meses (Fig. 4-a a Fig. 4-f). Para o trimestre MJJ essa defasagem cai para cinco meses. Os trimestres de primavera e parte do verão SON, OND, NDJ e DJF, também apresentam um desempenho razoável para previsões feitas com um e dois meses de antecedência, em alguns casos. A influência do Oceano Pacifico sozinho, na previsão, parece ser muito maior no final e início do ano e também para os trimestres iniciais da previsão. O desempenho do modelo cai bastante no período de inverno, próximo ao meio do ano.

\section{Grupo 3 - Oceano Pacífico e Atlântico Tropical como Preditores}

A Fig. 5(a-1) mostra os resultados do desempenho do modelo SIMOC quando utilizam-se os dois oceanos simultaneamente como campo preditor. Pelos resultados obtidos pode-se comparar e perceber que o desempenho do modelo que usou os dois oceanos juntos é muito similar àquele do modelo que usou somente o Oceano Pacífico. A inclusão dos dois oceanos em alguns casos parece prejudicar o bom desempenho da previsão. Este é o caso das previsões que partem no mês de janeiro (Fig. 5-a) e válidas nos trimestres iniciais. O Atlântico parece estar prejudicando o bom desempenho dessa previsão. Porém, pode-se perceber que em outros casos de previsões a inclusão de ambos oceanos tende a melhorar o desempenho geral do modelo, principalmente nos trimestres iniciais da previsões. Isto aconteceu por exemplo nos casos onde os meses preditores foram março, abril, maio e junho (Fig. 5-c a 5-f, respectivamente).

\section{UM ESTUDO DE CASO}

Neste item são apresentadas comparações entre as observações e as previsões elaboradas com o SIMOC. O modelo, nesse caso, foi construído com a base de dados do período de 1950 a 1980 e usando-se a TSM dos Oceanos Pacífico e Atlântico Tropical simultaneamente como campo preditor (Fig. 1a). O período escolhido para se avaliar essas previsões foi a primavera e o verão (austrais) do episódio ENOS de 1997-1998. São mostradas médias sazonais das anomalias de precipitação ocorridas em quatro trimestres no período de agosto de 1997 a janeiro de 1998, e as respectivas previsões feitas com defasagens variando de 1 a 3 meses (Fig. 6 a Fig. 9).

\section{Comparações das previsões com as observações no período de primavera e verão do episódio ENOS 1997-1998.}

A Fig. 6a mostra as anomalias observadas no trimestre agosto-setembro-outubro de 1997. As anomalias de precipitação ficaram acima da média climatológica, principalmente no noroeste do RS, centro e oeste de $\mathrm{SC}$ e sudoeste do PR. As Fig. 6b a 6d mostram as previsões feitas com o SIMOC para esse mesmo período (agosto-setembro-outubro de 1997), porém com o modelo sendo inicializado com condições iniciais diferentes (CI). A Fig. 6b é uma previsão feita com um mês de antecedência, onde usou-se a TSM de julho de 1997 para se prever o trimestre em questão. A Fig. $6 c$ também é uma previsão porém feita com dois meses de antecedência, usou-se a TSM de junho de 1997. A Fig. 6d é outra previsão porém com três meses de 


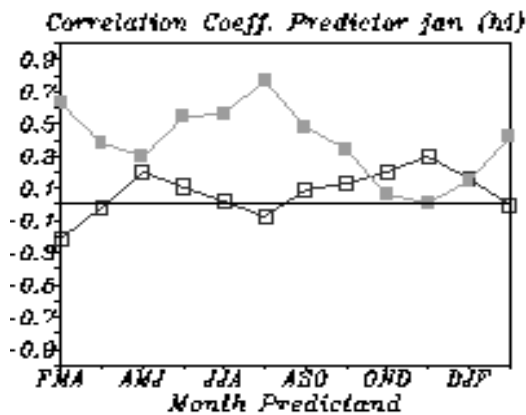

a)

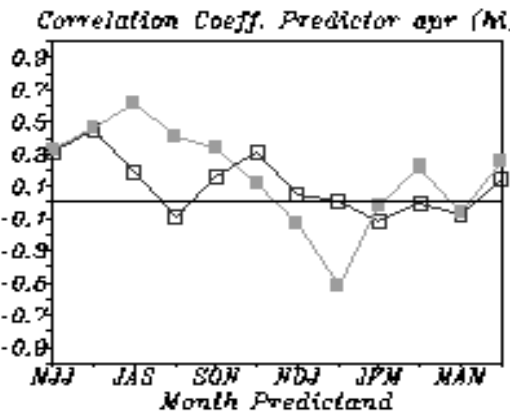

d)

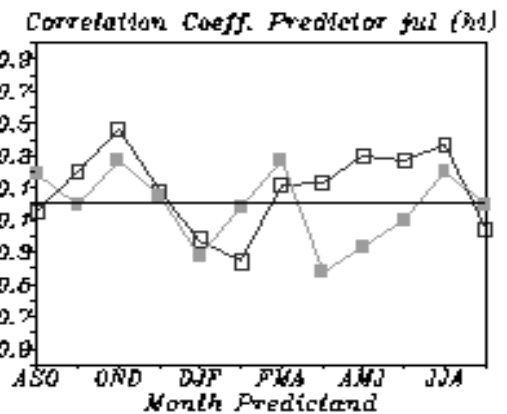

g)

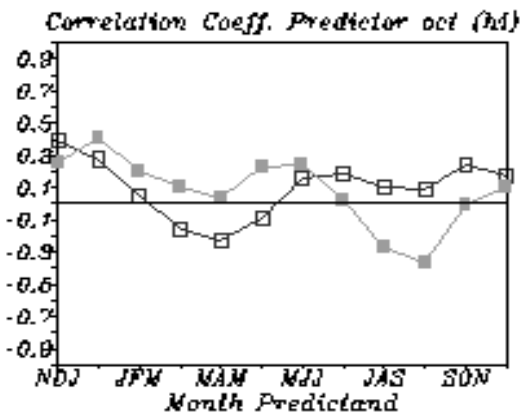

j)

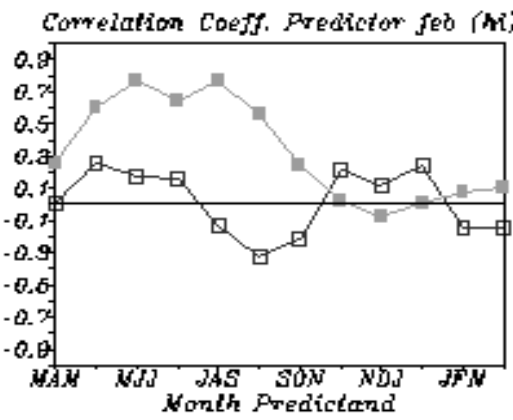

b)

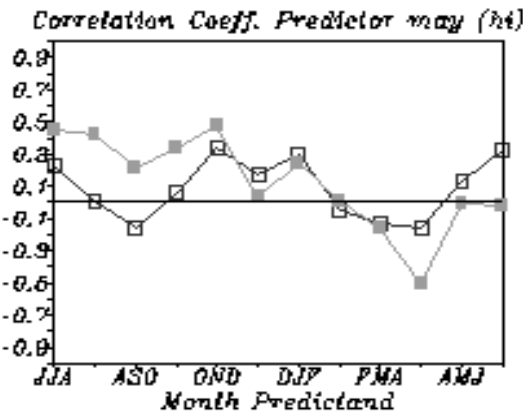

e)

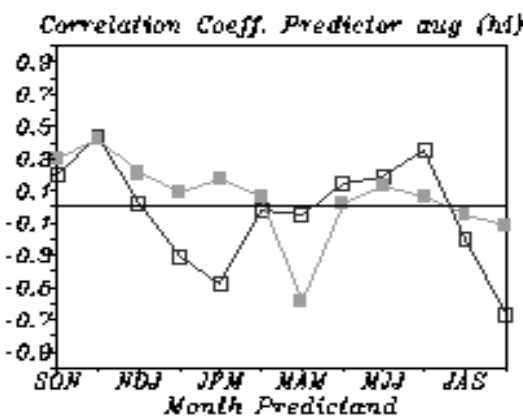

h)

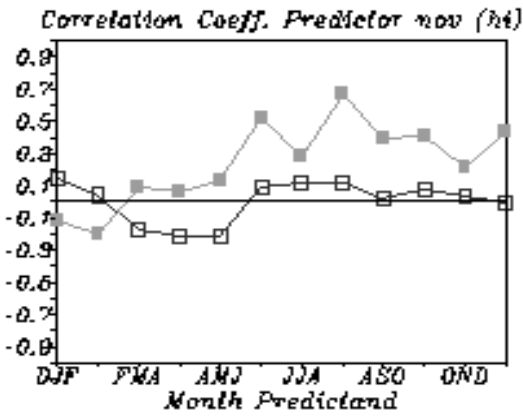

k)

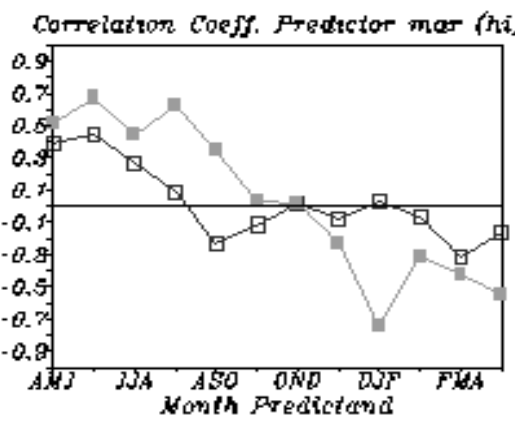

c)

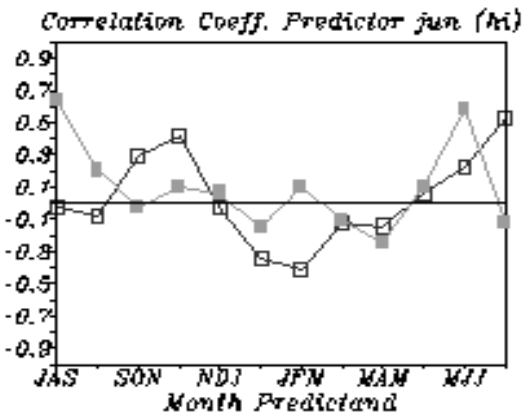

f)

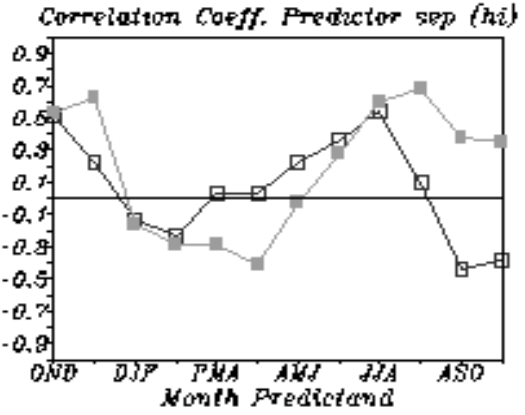

i)

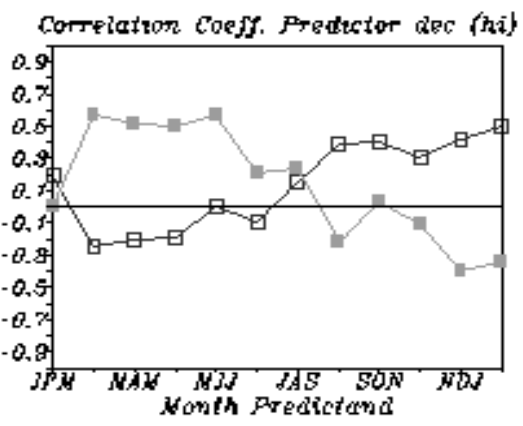

l)

Figura 5 - Coeficientes de correlação entre a persistência das anomalias de precipitação observadas com as anomalias observadas (símbolo aberto), e coeficientes de correlação entre as anomalias de precipitação previstas pelo SIMOC com as observadas (símbolo cheio), usando-se os Oceanos Pacífico e Atlântico Tropical como campo preditor. Começando com o experimento feito em Janeiro (5a) indo até Dezembro (51). Cada experimento tem 12 meses de defasagem.

Figure 5 - Correlation coefficients between observed precipitation anomalies and observed precipitation anomalies persistence (open symbols), and between forecasted precipitation anomalies and observed precipitation anomalies (full symbols), for SST in both Tropical Pacific and Atlantic Oceans as predictor fields. Initialization on the experiment starts from January (Fig. 5a) to December (Fig. 5l). Each experiment shown had a 12 month lag. 


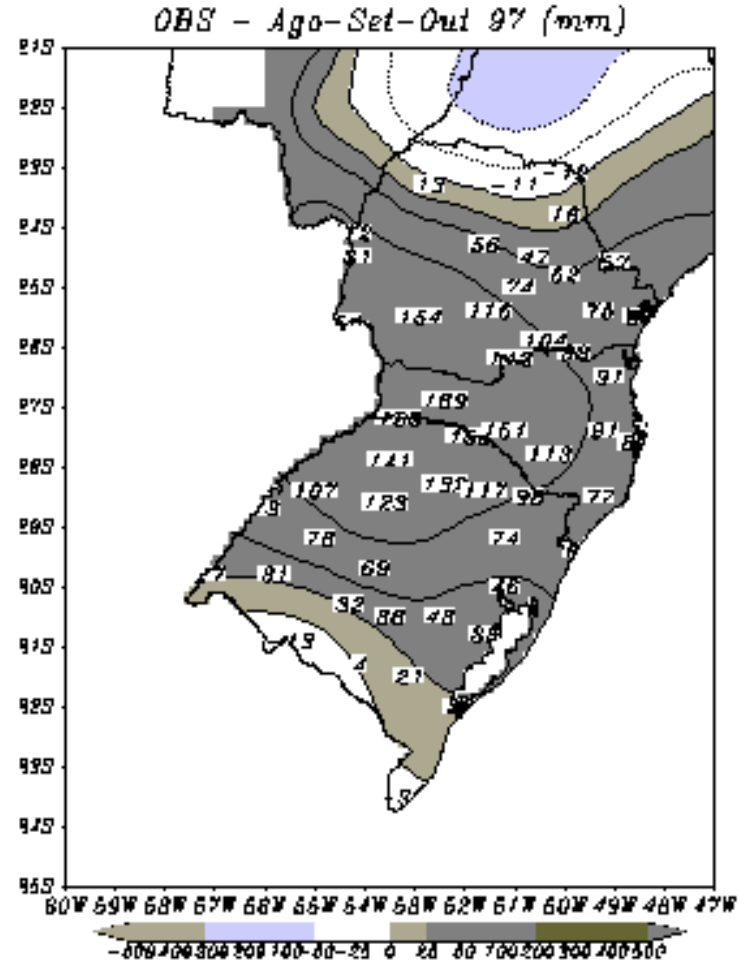

(a)

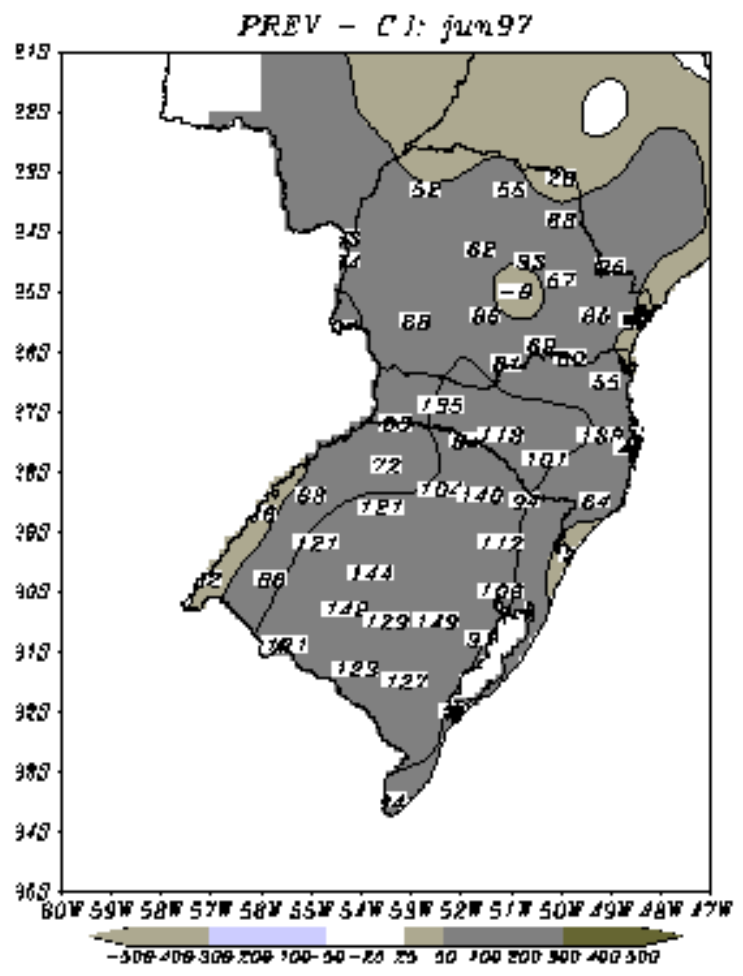

(c)

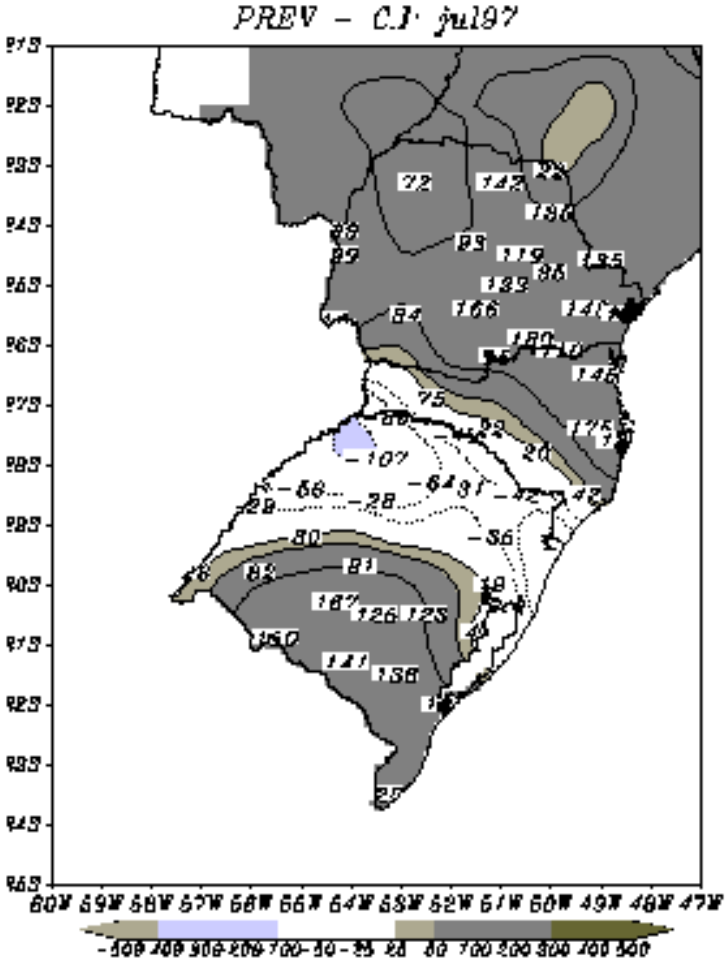

(b)

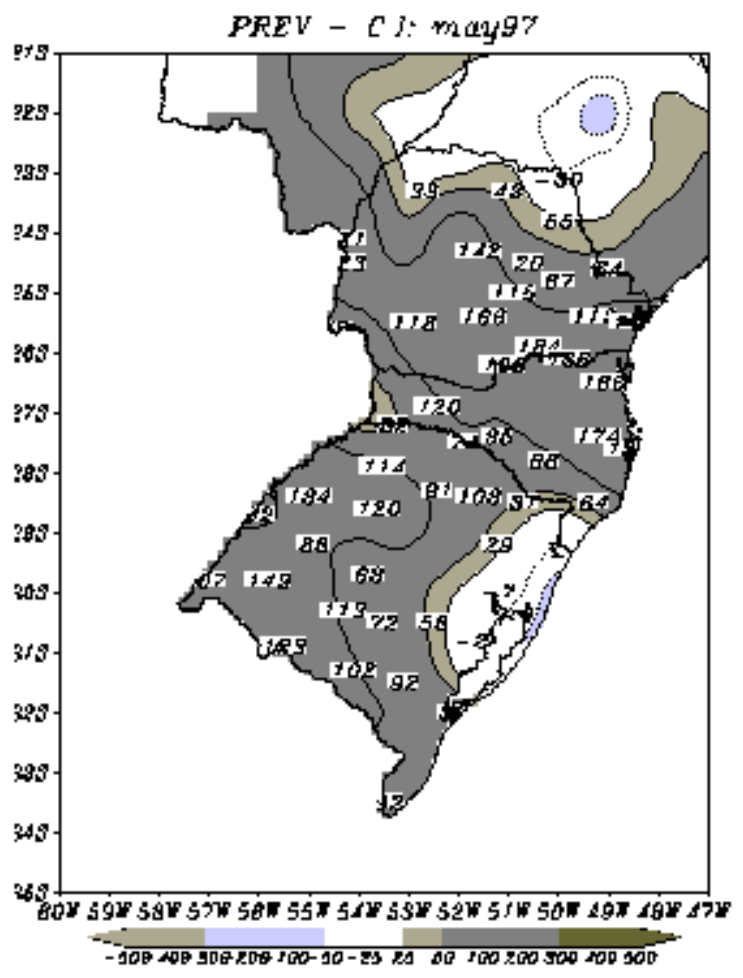

(d)

Figura 6 - Anomalias observadas de precipitação para a Região Sul do Brasil no trimestre Agosto-Setembro-Outubro-1997 (Fig. 6a). Previsões do SIMOC para o mesmo período, com 1 mês de antecedência (CI 07-1997) (Fig. 6b), 2 meses (CI 06-1997) (Fig. 6c) e 3 meses (CI 05-1997) (Fig. 6d).

Figure 6 - Observed precipitation anomalies over Southern Brazil during August-September-October-1997 (Fig. 6a). SIMOC's forecasts for the same period, performed one month before (Initial Conditions IC 07-1997) (Fig. 6b), 2 months before (IC 06-1997) (Fig. 6c) and 3 months(IC 05-1997) (Fig. 6d). 


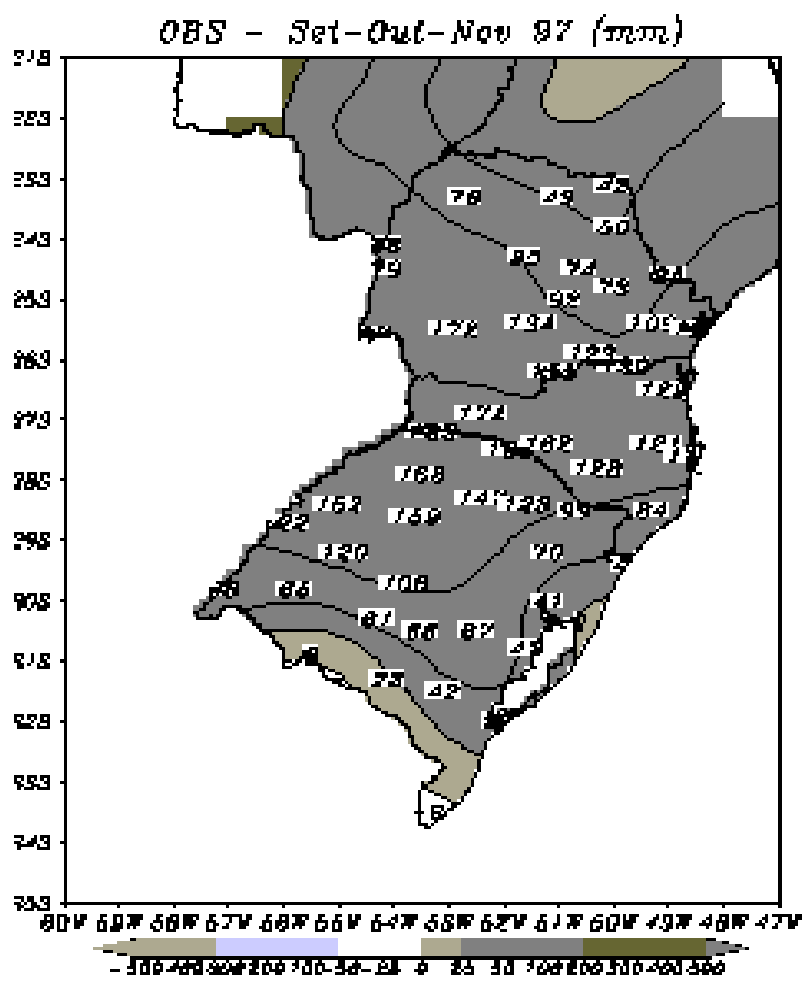

(a)

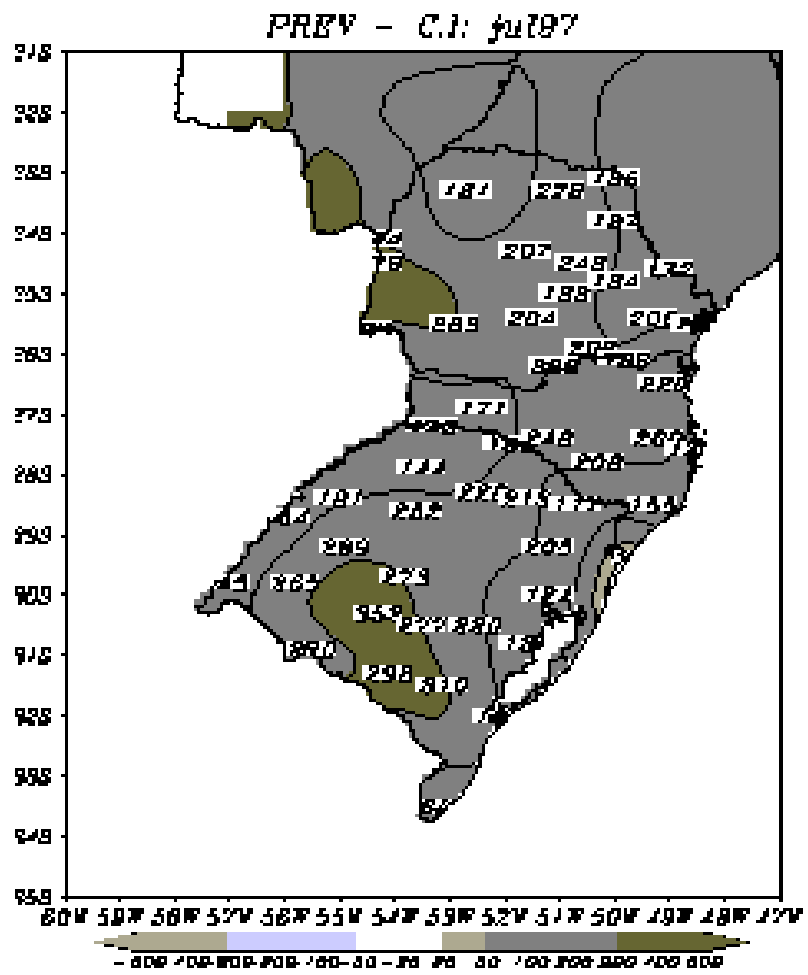

(c)

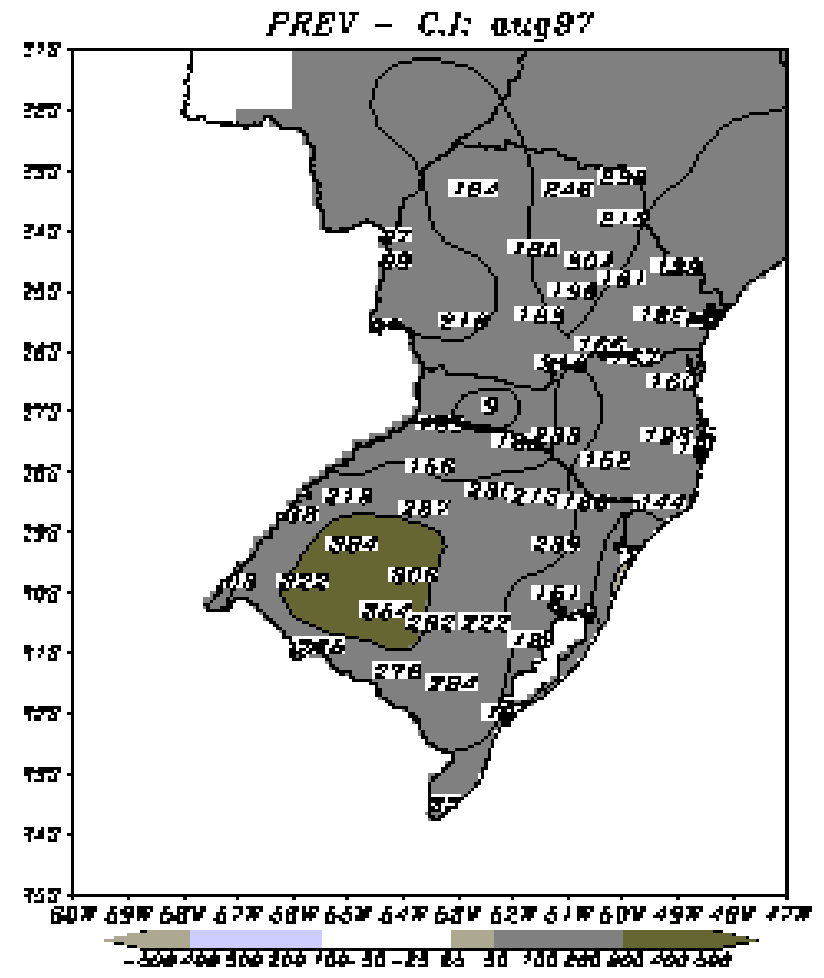

(b)

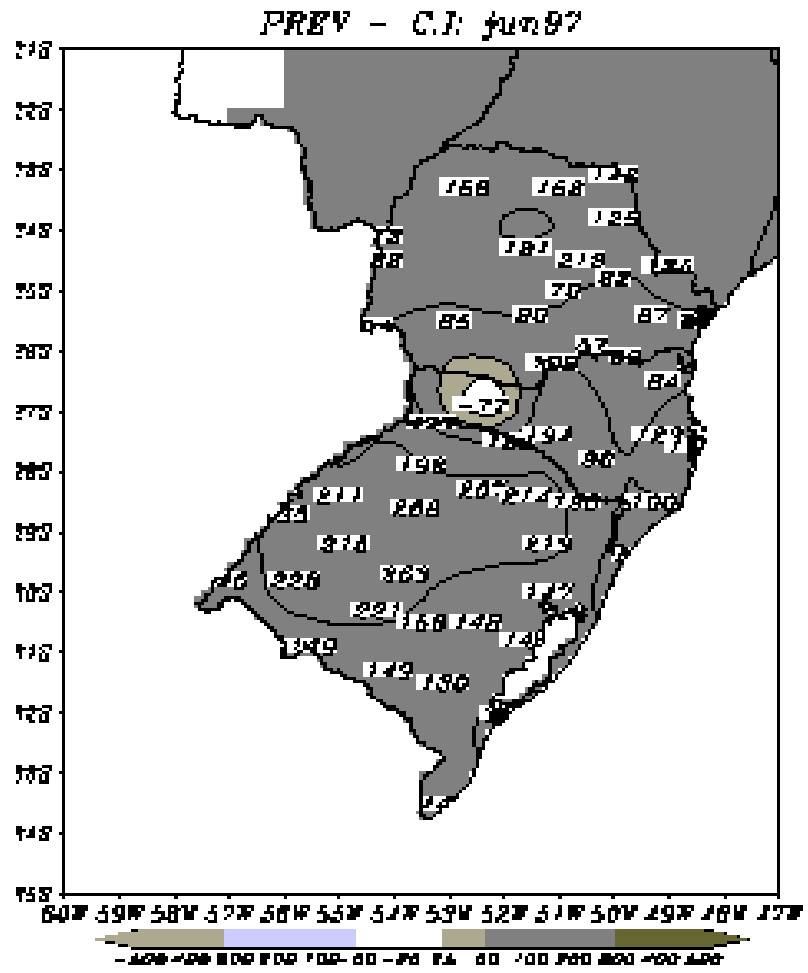

(a)

Fig. 7 - Anomalias observadas de precipitação para a Região Sul do Brasil no trimestre Setembro-Outubro-Novembro-1997 (7a). Previsões do SIMOC para o mesmo período, com 1 mês de antecedência (CI 08-1997) (7b), 2 meses (CI 07-1997) (7c) e 3 meses (CI 06-1997) (7d).

Figure 7 - Observed precipitation anomalies over Southern Brazil during September-October-November-1997 (Fig. 7 a). SIMOC's forecasts for the same period, performed one month before (IC 08-1997) (Fig. 7b), 2 months before (IC 07-1997) (Fig. 7c) and 3 months(IC 06-1997) (Fig. 7d). 
antecedência (TSM de maio de 1997). Pode-se perceber que as previsões feitas com 2 e 3 meses de antecedência mostraram melhores resultados, quando comparadas com as observações do que a previsão realizada apenas com um mês de antecedência. De um modo geral as previsões, vistas nas Fig. $6 \mathrm{c}$ e $6 \mathrm{~d}$, foram capazes de reproduzir o sinal correto da observação, mostrando anomalias de precipitação acima da média climatológica em quase toda a região.

O trimestre setembro-outubro-novembro 1997 (Fig. 7a), mantém o mesmo padrão de anomalias observadas do período anterior, porém com a área de anomalias máximas (superiores a $100 \mathrm{~mm}$ ) aumentada em extensão. Nesse caso, as previsões feitas de 1 a 3 meses de antecedência (Fig. $7 b$ a Fig. $7 d$ ) reproduzem o padrão das anomalias observadas um pouco melhor do que o trimestre anterior, em relação ao sinal da anomalia. Todas as previsões apontam para o excesso de precipitação durante o trimestre analisado e de um modo geral, os valores previstos superestimam as observações.

Em outubro-novembro-dezembro de 1997 (Fig. 8a) ocorreram as maiores anomalias positivas de precipitação de todo o período analisado, com anomalias superiores a $100 \mathrm{~mm}$ em praticamente toda a Região Sul, exceto no norte do PR. Uma área com máximos superiores a $200 \mathrm{~mm}$ concentrou-se no noroeste do RS. Esse quadro excessivamente anômalo causou sérios danos ao oeste do RS deixando várias cidades inundadas. Nesse período acontecem os maiores impactos do ENOS no sul do Brasil durante o seu ano de surgimento. As Fig. 8b a Fig. 8d mostram que o modelo foi capaz de prever o quadro observado, com uma antecedência de até três meses. Em todos os casos, o modelo reproduziu o mesmo sinal das anomalias observadas. Nesse caso, além do sinal, o modelo também indicou uma magnitude de anomalias similares àquelas ocorridas sobre o noroeste do RS (local onde ocorreram os maiores valores de precipitação) com valores em torno dos $200 \mathrm{~mm}$ e com 1 e 2 meses de antecedência (Fig. 7b e Fig. 7c).

No período de novembro-dezembro-janeiro de 98 (Fig. 9a a Fig. 9d), as anomalias ficaram acima da média climatológica em toda a área (superiores a $100 \mathrm{~mm}$ ), com a porção mais ao sul do RS apresentando os menores valores (entre $50 \mathrm{~mm}$ e $100 \mathrm{~mm}$.). As previsões para esse período apresentaram um melhor desempenho para a porção central da Região Sul (sul do $\mathrm{PR}, \mathrm{SC}$ e norte do RS). A previsão feita com a TSM de outubro de 1997 (um mês de antecedência) apresentou o melhor desempenho. Nas demais áreas, o modelo não obteve um bom desempenho.

\section{CONCLUSÕES}

Nesse trabalho foram apresentados os resultados de um modelo regional estatístico baseado no método de Análise de Correlações Canônicas (ACC). O modelo prevê anomalias de precipitação trimestrais para a Região Sul do Brasil (PR, SC e RS) e usa como campo preditor as anomalias mensais observadas de TSM dos Oceanos Atlântico e Pacífico. Na primeira parte dos resultados apresentou-se uma visão geral do desempenho global do modelo usando-se três áreas de TSM sobre os Oceanos Pacífico e Atlântico como campo preditor. No caso onde usou-se o Oceano Atlântico como preditor, esse oceano pareceu influenciar no bom desempenho das previsões a medida em que a previsão afastou-se em relação ao seu mês preditor. Esse comportamento ficou claro em vários meses, principalmente no período próximo ao final e início do ano. No caso do Oceano Pacífico, esse oceano pareceu ter uma influência maior no início da previsão apresentando um desempenho maior nos primeiros seis meses de previsão, ao contrário do caso anterior. Como no caso do Atlântico, esse caso também mostrou que os melhores desempenhos do modelo ocorreram no final e início do ano. Para o caso onde se usou ambos os oceanos Pacífico e Atlântico simultaneamente os desempenhos são prejudicados, em alguns casos. Porém, de uma maneira geral o uso dos dois oceanos tende a melhorar o desempenho das previsões.

A análise dos resultados de previsão para o episódio ENOS 1997-1998 mostrou que o modelo reproduziu as anomalias observadas de um modo geral, acertando as previsões principalmente em relação ao sinal da anomalia de precipitação observada. O período em que o modelo apresentou os melhores resultados foi no trimestre outubro-novembro-dezembro de 1997. Nesse trimestre, o modelo conseguiu reproduzir o sinal das anomalias observadas (Fig. 7b a 7d) e também magnitude aproximada (Fig. 7b). Uma provável hipótese para o maior sucesso dessa previsão é a de que nesse período ocorrem os maiores impactos do ENOS em seu ano de surgimento. Em particular, no ano de 1997, verificou-se que as anomalias de precipitação ficaram acima da média em toda a região, e também foram as maiores anomalias ocorridas em todo o período analisado.

O exercício de previsão e comparação com as observações para a Região Sul, no período de primavera e início de verão no ano de 1997, mostrou que o modelo regional estatístico apresenta uma boa potencialidade para ser usado na previsão sazonal dessa região. Porém, essa potencialidade de previsão deve 

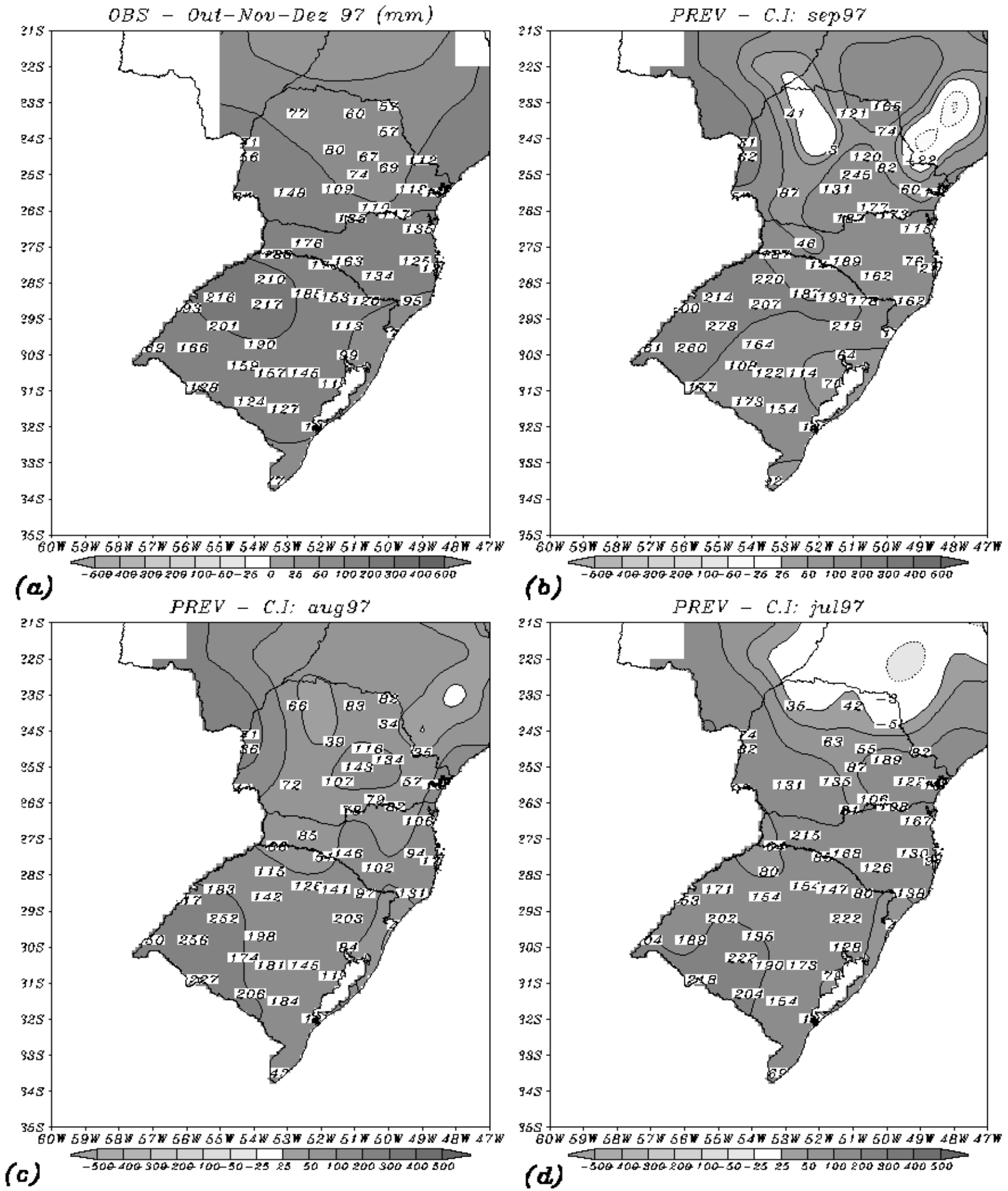

Figura 8 - Anomalias observadas de precipitação para a Região Sul do Brasil no trimestre Outubro-Novembro-Dezembro-1997 (Fig. 8a). Previsões do SIMOC para o mesmo período, com 1 mês de antecedência (CI 09-1997) (Fig. 8b), 2 meses (CI 08-1997) (Fig. 8c) e 3 meses (CI 07-1997) (Fig. 8d).

Figure 8 - Observed precipitation anomalies over Southern Brazil during October-November-December-1997 (Fig. 8a). SIMOC's forecasts for the same period, performed one month before (IC 09-1997) (Fig. 8b), 2 months before (IC 08-1997) (Fig. 8c) and 3 months(IC 07-1997) (Fig. 8d). 


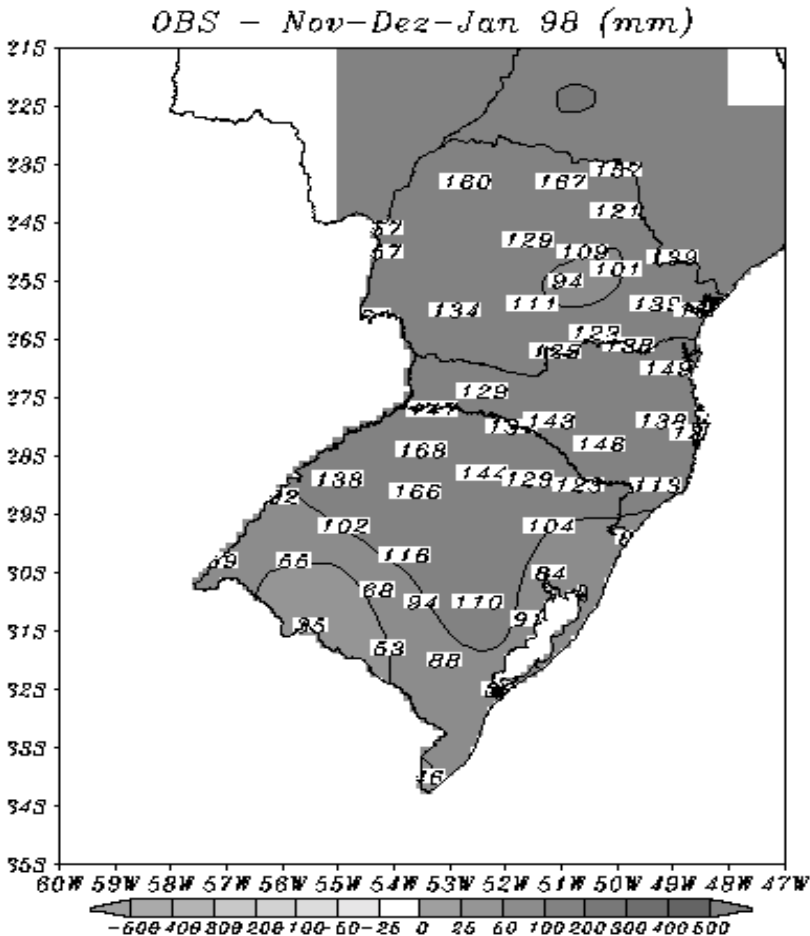

(a)

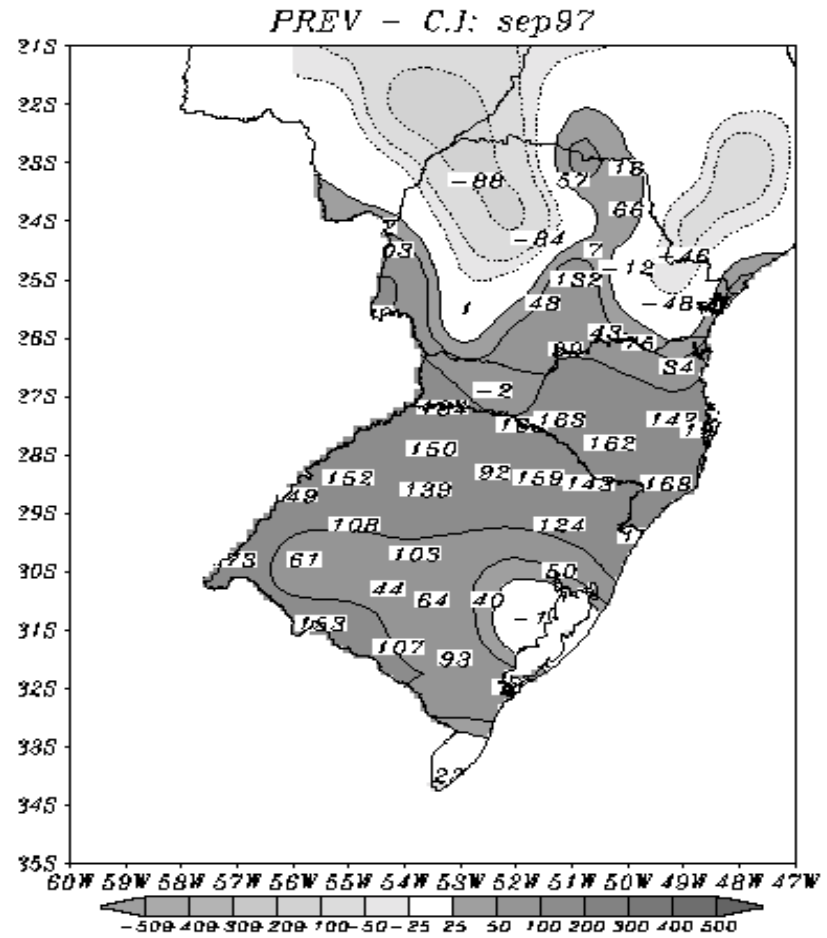

(c)

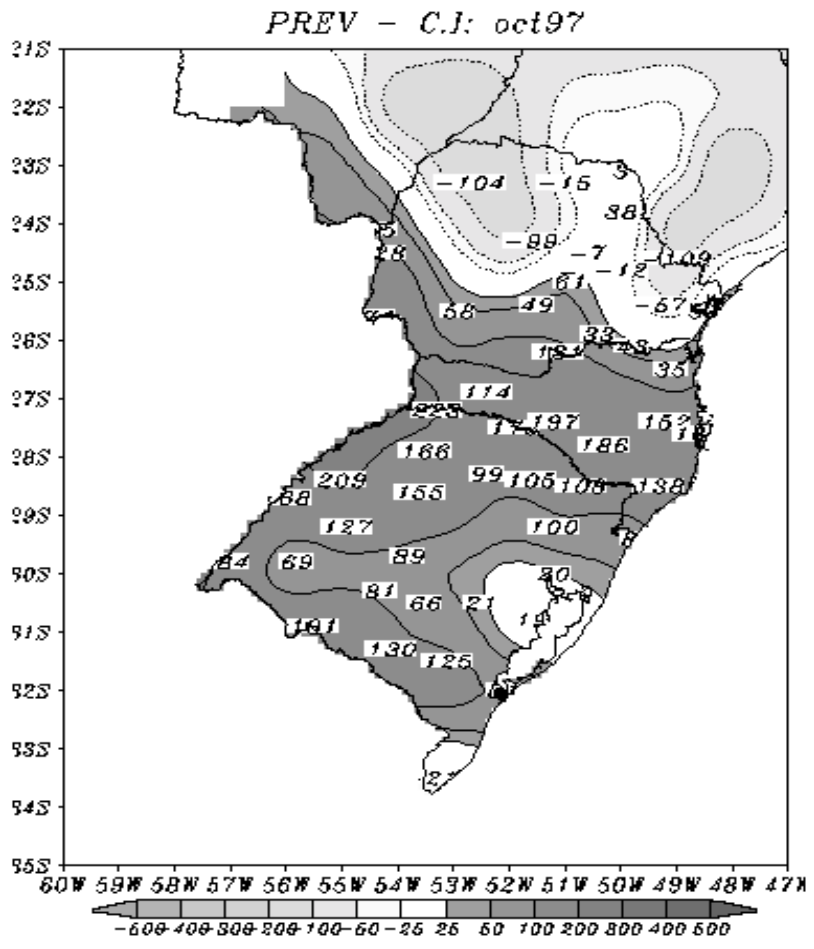

(b)

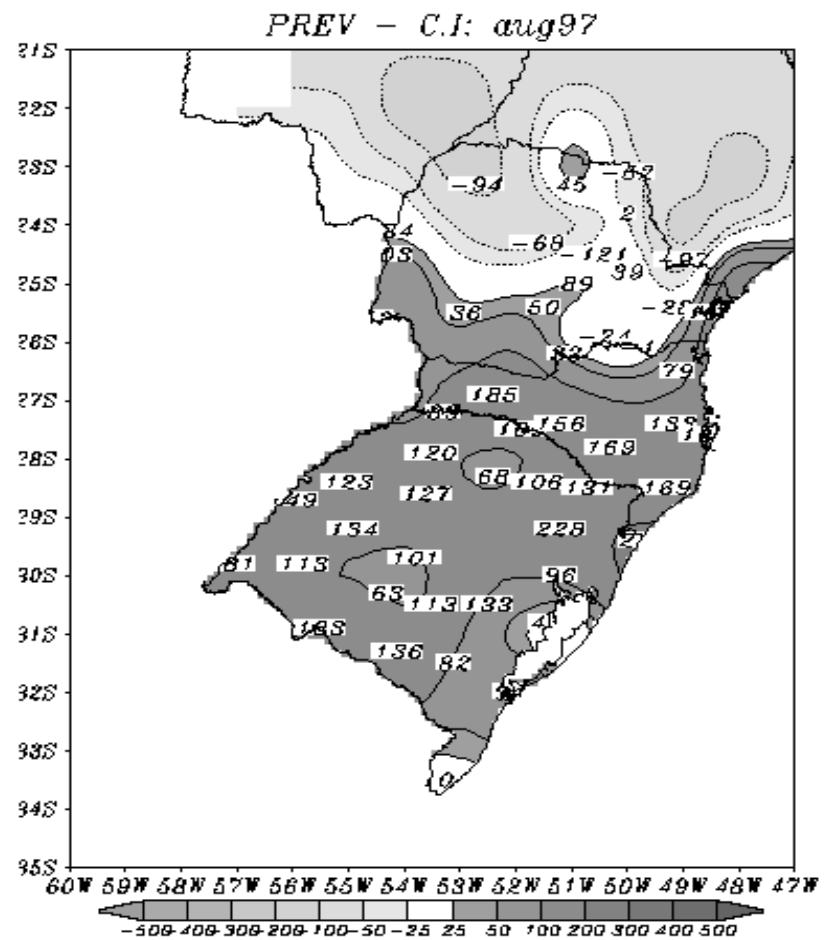

(d)

Figura 9 - Anomalias observadas de precipitação para a Região Sul do Brasil no trimestre Novembro-Dezembro-1997-Janeiro/98 (Fig. 9a). Previsões do SIMOC para o mesmo período, com 1 mês de antecedência (CI 10-1997) (Fig. 9b), 2 meses (CI 09-1997) (Fig. 9c) e 3 meses (CI 08-1997) (Fig. 9d).

Figure 9 - Observed precipitation anomalies over Southern Brazil during November-December-1997-January/98 (Fig. 9a). SIMOC's forecasts for the same period, performed one month before (IC 10-1997) (Fig. 9b), 2 months before (IC 09-1997) (Fig. 9c) and 3 months(IC 08-1997) (Fig. 9d). 
ser explorada para outras situações climáticas, bem como ser estendida para outros períodos do ano.

Um ponto interessante a ser buscado em estudos futuros é o melhor entendimento do sistema de acoplamento oceano-atmosfera e suas possíveis influencias na circulação atmosférica e precipitação na Região Sul do Brasil. Isso poderia ajudar a elucidar o papel dos oceanos Atlântico e Pacífico nas previsões sazonais e variabilidade climática dessa região.

\section{AGRADECIMENTOS}

Os autores agradecem ao $8^{\circ}$ Distrito de Meteorologia ( $8^{\circ} \mathrm{DISME} /$ INMET), Departamento Nacional de Energia Elétrica (DNAEE), Instituto de Pesquisas Agronômicas (FEPAGRO), Companhia Paranaense de Energia Elétrica (COPEL), Sistema Meteorológico do Paraná (SIMEPAR) e ITAIPU Bi-Nacional, pelo fornecimento dos dados de precipitação. Agradecem também a Carlos A. Nobre, Pedro L. S. Dias, José Marengo, Iracema F. A. Cavalcanti, Denise Fontana e Moacir Berlato pelo auxílio, incentivo, críticas e sugestões na adaptação e desenvolvimento das rotinas computacionais do SIMOC para a Região Sul do Brasil bem como nas discussões durante a elaboração desse manuscrito. Ao Grupo de Clima pela operação do SIMOC nas rotinas de previsão sazonal climática realizadas no CPTEC/INPE. Aos revisores anônimos pelas críticas construtivas durante a fase de revisão desse trabalho. E também a todos aqueles que de alguma maneira colaboraram com esse projeto.

\section{REFERÊNCIAS}

ACEITUNO, P. - 1988 - On the functioning of the Southern Oscillation in the South American Sector. Mon. Wea. Rev., 116: 505-524.

BARNETT, T. P. \& PREISENDORFER, R. - 1987 - Origins and levels of monthly and seasonal forecast skill for United States surface air temperatures determined by canonical correlation analysis. Mon. Wea. Rev., 115:1825-1849.

BARNSTON, A. G. - 1994 - Linear Statistical shortterm climate predictive skill in the Northern Hemisphere. J. Climate, 7: 1513-1564.

BARNSTON, A. G. \& ROPELEWSKI, C. F 1992 - Prediction of ENSO episodes using canonical correlation analyses. J. Climate, 5: 13161345.

BARNSTON, A. G. \& SMITH, T. M. - 1996 Specification and prediction of global surface temperature and precipitation from global SST using CCA. J. Climate, 9: 2660-2697.

BENDAT, J.S., \& PERSOL, A.G. - 1986 - Random Data - Analysis and measurement procedures, 2nd. Edition, John Wiley \& Sons, NY, 525 pp.

BERLATO, M. A. - 1992 - The climate forecasting applications in the decision-making process for the southern region of Brazil. In: Workshop report ENSO and seasonal to interannual climate variability, socio-economic impacts, forecasting and applications to the decision making process. 14-16 de Setembro. Florianopolis, Brasil

BERLATO, M. A. \& FONTANA, D. S. - 1997 El Niño Oscilação Sul e a Agricultura da Região Sul do Brasil. Workshop and Conference on the 1997-98 El Niño Impacts and Potential Applications for climate Forecasts in Southeast South America. December 10-12. Montevideo, Uruguay.

DIAZ, A. F., STUDZINSKI, C. D. \& MECHOSO, C. R. - 1998 - Relationships between Precipitation Anomalies in Uruguay and Southern Brazil and Sea Surface Temperature in the Pacific and Atlantic Oceans. J. Climate. 11: 251-271.

GHIETTO, M. A. - 1998 - Pronóstico de anomalías de preicpitación a la Región Sur de Sudamérica: Pronóstico de anomalías de Precipitación y Temperatura Relatório técnico das atividades exercidas como pesquisadora visitante no INPE/CPTEC. Proyecto IAI-WMO-REF-RLA/92/G34. Cachoeira Paulista - SP.

GRAHAM, N. E., MICHAELSEN, J. \& BARNETT, T. P. - 1987a - An Investigation of El Niño-Southern Oscillation cycle with statistical models. Part 1: Predictor field characteristics. J. Geophys. Res. 92:14251-14270.

GRAHAM, N. E., MICHAELSEN, J. \& BARNETT, T. P. - 1987b - An Investigation of El Niño-Southern Oscillation cycle with statistical models. Part 2: Model results. J. Geophys. Res. 92:14271-14289.

GRIMM, A. M., TELEGINSKI, S. E., FREITAS, E. D.,COSTA, S. M. S., FERLIZI, P. G. \& GOMES, J. - 1996 - Anomalias de precipitação no Sul do Brasil em eventos El Niño. Anais do IX Congresso Brasileiro de Meteorologia, Sociedade Brasileira de Meteorologia, Campos do Jordão SP, 2:1098-1102.

GRIMM, A., FERRAZ, A. \& GOMES, J. -1998 Precipitation anomalies in Southern Brazil associated with El Nino and La Nina events. J. Climate, 11: 2863-2880.

MARENGO, J. - 1995 - Variations and change in South American streamflow. Climate Change, 31: 99-117.

PEZZI, L. P. - 1998 - Previsões Regionais Sazonais e Comparações com Observações para o Sul do Brasil durante o Episódio El Niño Oscilação Sul 97/98. Workshop sobre o El Niño1997/98: Previsões, Impactos e Lições para o Futuro. Anais 
do X CBMet e VII FLISMET. Brasília - DF EN98014.

PEZZI, L. P., REPELLI, C. A., NOBRE, P., CAVALCANTI, I. F. A. \& SAMPAIO, G. 1998 - Forecasts of Tropical Atlantic SST Anomalies using a Statistical Ocean Model at CPTEC/INPE - Brazil. Exp. Lon. Lead For. Bull, 7.

RAO, V. B. \& HADA K., - 1990 - Characteristics of rainfall over Brazil: Annual variations and connections with the Southern Oscillation. Theor. Appl. Climatol., 42: 81-91.

REPELLI, C. A. - 1995 - Sistema de Modelagem Estatística das Temperaturas da Superfície do Mar - SIMOC.- Relatório técnico de atividades exercidas como pesquisador visitante no INPE/ CPTEC apoiado pelo CNPq. Cachoeira Paulista $-\mathrm{SP}$.

REPELLI, C. A. \& NOBRE, P. - 2000 - Statistical Prediction of Sea Surface Temperature over the Tropical Atlantic. Submitted and Accepted to the International Journal of Climatology.

REYNOLDS, R. W. \& SMITH T. M - 1995 - A high-resolution global sea surface temperature climatology. J. Climate, 8: 1571-1583.

ROJAS, M. I.- 1997 - Aplicación del Sistema de Modelaje Estadístico de Los Oceanos - Simoc en el estudio de las anomalias de precipitación en Venezuela. Relatório técnico das atividades exercidas como pesquisadora visitante no INPE/CPTEC. Proyecto IAI-WMO-GEF-RLA/92/G34. Out. 1997. Cachoeira Paulista - SP.

ROPELEWSKI, C. F. \& HALPERT, M. S. - 1987 - Global and regional scale precipitation patterns associated with El Nino/Southern Oscillation. Mon. Wea. Rev., 115:1606-1626.

ROPELEWSKI, C. F. \& HALPERT, M. S. - 1989

- Precipitation patterns associated with the high index phase of the Southern Oscillation. J. Climate, 2: $268-284$

SMITH, T. M., REYNOLDS, R. W., LIVEZEY, R. E., STOKES, D. C. - 1996 - Reconstruction of historical Sea Surface Temperatures using empirical Orthogonal Functions. J. Climate, 9: 1403-1420.

STUDZINSKI, C. D. - 1995 - Um estudo da precipitação na Região Sul do Brasil e sua Relação com os Oceanos Pacífico e Atlântico Tropical Sul. São José dos Campos. INPE. Dissertação de Mestrado em Meteorologia, 87pp

UBARANA, V. N.- 1996 - Adaptação do Sistema de Modelagem Estatítica dos Oceanos - Simoc - para o prognóstico de anomalias de precipitação. Verificação para a Região Norte do Nordeste do Brasil. Relatório técnico das atividades exercidas como pesquisador visitante no INPE/CPTEC. Processo nr.460494/96-1. Cachoeira Paulista - SP.

\section{NOTE ABOUT THE AUTHORS}

\section{Luciano Ponzi Pezzi}

Formou-se em meteorologia no ano de 1989, na Universidade Federal de Pelotas (UFPel). Em 1993, recebeu o título de Mestre em Sensoriamento Remoto no Centro Estadual de Pesquisas em Sensoriamento Remoto e Meteorologia (CEPSRM-UFRGS). Desde de 1993, trabalha no CPTEC-INPE onde tem desenvolvido trabalhos de pesquisa na área de variabilidade e modelagem climática, usando modelos estatísticos e dinâmicos. Participou de cursos na Alemanha (GKSS) e na Itália (ICTP) nas áreas de mudanças climáticas globais e regionais. Foi pesquisador visitante no ano de 1996 no NCEPNOAA (EUA) e em 1998 no LMD (Universidade de Paris). Coordenou a execução da previsão climática para o evento ENOS 1997-1998 usando a Técnica de Previsão por Conjuntos realizada com um elevado número de integrações do modelo climático CPTEC-COLA. Atualmente está cursando o doutorado em Oceanografia Física na School of Ocean and Earth Sciences (SOESSOC), University of Southampton, United Kingdom.

\section{Vinicius Ubarana}

Físico, de formação, pela Universidade Estadual Paulista UNESP (1990). Recebeu o título de Mestre em Meteorologia pelo Instituto Nacional de Pesquisas Espaciais - INPE na área de micrometeorologia, em 1993. Trabalhou com modelagem do ciclo hidrológico até 1994, no Brasil e Inglaterra, passando, então, a área atual de previsão de tempo e clima. Desenvolveu várias atividades operacionais e de pesquisa científica, em modelagem estatística e previsibilidade de tempo e clima, no INPE e na Fundação Cearense de Meteorologia e Recursos Hídricos - FUNCEME. Trabalha atualmente no The Weather Channel (EUA) como meteorologista sênior responsável pela previsão de tempo para toda a América Latina, além de atividades em câmera como expert em previsão.

\section{Carlos Repelli}

Bacharel em Física pela Universidade Estadual PaulistaUNESP (1987) mestre em Meteorologia (área de modelagem numérica) pelo Instituto Nacional de Pesquisas Espaciais-INPE, (1990). Entre 1991 e 1997 foi pesquisador da FUNCEME, onde desenvolveu vários projetos sobre o clima da Região Nordeste, sua modelagem e previsibilidade. Durante este período fez cursos na Itália (ICTP) e Estados Unidos (NWS e IRI) relacionados à modelagem dinâmica e estatística do clima e dos oceanos tropicais. Foi pesquisador visitante do CPTEC-INPE onde desenvolveu o primeiro modelo operacional de previsão sazonal das temperaturas da superfície do mar sobre o Attântico Tropical, contribuindo para melhorar as previsões sazonais climáticas de todo o Brasil. Iniciou seu doutorado em Oceanografia Física na Columbia University, New York em 1997. Atualmente é Coordenador de Produtos do The Weather Channel Latin America, onde trabalha no desenvolvimento de ferramentas para a previsão do tempo operacional. 


\section{FORECAST AND PERFORMANCE OF A STATISTICAL REGIONAL MODEL FOR SEASONAL CLIMATE PREDICTION IN SOUTHERN BRAZIL}

Seasonal and interannual climate variability affect agricultural activities in Southern Brazil, with consequences on production and the economy. On interannual time scales, especially those related to El Niño or La Niña cycles, the occurrence of extreme events such as drought or flood has shown the sensitivity of agricultural activities to climate variability. There is therefore a need for detailed seasonal climate forecasts in order to minimise the impacts of climate hazards on agriculture. In Southern Brazil, the winter wheat is the main crop that is affected by climate hazards. This has motivated the development of dynamical and statistical seasonal climate forecasts. One such model developed on statistical techniques is based on Canonical Correlation Analysis. This model is called SIMOC (Oceanic Modelling Statistical System, or SIstema de Modelagem Estatistica dos OCeanos) and it has been adapted to forecast seasonal rainfall anomalies. The predictors for this model are sea surface temperature in regions of the Pacific and the Atlantic Oceans, and the output is precipitation. As a predictor, the Atlantic Ocean seems to affect more the prediction in the correlation analysis when the lag increases. Considering the Pacific Ocean, the best forecast were obtained when the lag was smaller, at the beginning of the run, in opposite when the Atlantic Ocean was considered. In general, the use of the two oceans together improves the precision of the predictions compared to individual ocean cases. For the ENSO episode 1997-1998, the model succesfully reproduced the observed rainfall anomalies. The results discussed here indicate the great potential of the SIMOC model in predicting seasonal climate rainfall anomalies, particulary on spring and summer, as well as for the other seasons. Based on these discussions, further studies are being developed at CPTEC to better understand the coupling atmosphere-ocean system, and the possible influences of this coupling and its variations on circulation and precipitation in the region. It is important to identify the relative role of the Atlantic and Pacific Oceans and the interaction of both oceans on the seasonal climate in Southern Brazil and its variability.

\section{INSTITUTO NACIONAL DE PESQUISAS ESPACIAIS PÓS-GRADUAÇÃO EM METEOROLOGIA}

O Curso de Pós-Graduação em Meteorologia do INPE, cujo objetivo é a formação de pessoal a níveis de mestrado e doutorado, é reconhecidamente conceituado no Brasil e no exterior, contando com um corpo docente altamente qualificado, (24 doutores) atuante em linhas de pesquisas nas diferentes áreas da Meteorologia e da Interação Oceano-Atmosfera. Por outro lado, o INPE mantém um ativo programa de intercâmbio de pessoal técnico-científico através de convênios assinados com várias instituições similares no Brasil e no exterior, além de participar de experimentos científicos nacionais e internacionais.

Além dos suportes oferecidos pelos órgãos que fomentam a Pós-Graduação (CAPES, CNPq, FAPESP, e outros), os alunos usufruem de várias facilidades oferecidas pela própria Instituição.

Em regime de tempo integral, o tempo médio para cumprir o programa de Mestrado é de 24 meses, enquanto que para obter o título de Doutor é de 48 meses. Os períodos letivos são trimestrais, com férias acadêmicas em janeiro.

O Curso de Pós-Graduação em Meteorologia obteve conceito 4, tanto no Mestrado quanto no Doutorado, na última avaliação da CAPES, realizada em 1998. 


\title{
SEG/EAGE Distinguished Instructor Short Course
}

\author{
Seismic Amplitude Interpretation \\ By Fred Hilterman \\ 26 March 2002 \\ SOUTH AMERICAN COPACABANA HOTEL \\ Rua Francisco Sá, 90 - Copacabana \\ Rio de Janeiro
}

During the last 30 years, seismic interpreters have routinely applied bright spot and AVO technology for recognizing prospects and predicting lithology. New amplitude attributes were added to this technology as new exploration problems were defined. $R \& D$ continues in the field of amplitude interpretation, especially as $E \& P$ costs escalate as more severe environments are explored, such as the ultra-deepwater plays. With the high interest in reducing exploration risk, this

course addresses the methodology of an amplitude interpretation and the subsequent benefits and limitations that one can expect in various rock-property settings.

The first part of the course reviews relationships between rock properties and geophysical observations. Practical problems illustrate the assumptions and limitations of commonly used empirical transforms. In addition, step-by-step procedures for conducting and verifying fluid-substitution techniques are presented.

The second part identifies the components of the seismic response that are best suited for differentiating pore-fluid from lithologic effects. Field examples emphasize what combination of seismic signatures should be expected for different rock-property environments.

The third part provides rules of thumb for predicting AVO responses and interpreting lithology from observed responses. These rules of thumb help select the best seismic attribute for calibrating amplitude to rock properties in different areas. A case history illustrates the rock-property calibrations that are needed.

The last part examines the numerous amplitude attributes that can be extracted from seismic to quantify an interpretation. The benefits and limitations of these attributes in soft to hard-rock environments are discussed first with model data and then with case histories.

Who Should Attend?

This is a good opportunity for those interested in solving practical problems involving seismic amplitude interpretation. An excellent reference book is provided to the participants.

Instructor Biography:

Fred J. Hilterman received a geophysical engineering degree and $\mathrm{PhD}$ in geophysics from the Colorado School of Mines. During his tenure with Mobil (1963-1973), his assignments ranged from field work and prospect evaluation to Activity Leader at the Field Research Laboratory. In 1973, he joined the University of Houston as a Professor of Geophysics. While at UH, Fred co-founded the Seismic Acoustics Laboratory (SAL) and was Principal Investigator until 1981. At that time, he co-founded Geophysical Development Corporation where he is currently Vice-President of Development. Fred also lectures at UH as a Distinguished Research Professor. Fred is a long-standing member of SEG, EAGE, and AAPG, with honorary memberships in SEG and GSH. His services to the societies include Associate Editor of GEOPHYSICS, Chairman of TLE Editorial Board, SEG and AAPG Distinguished Lecturer, and both Technical and General Chairman of SEG Annual Meetings. Fred received the SEG Best Paper Award, Best Presentation Award, and Virgil Kauffman Gold Medal. He has been an instructor for SEG and AAPG Continuing Education courses since the 1970s. Fred was the 1996-97 SEG President. 